\title{
INTEGRABILITY OF GEODESIC FLOWS FOR METRICS ON SUBORBITS OF THE ADJOINT ORBITS OF COMPACT GROUPS
}

\author{
IHOR V. MYKYTYUK*
}

\author{
Institute of Mathematics \\ Cracow University of Technology \\ Warszawska 24 \\ 31155, Cracow, Poland \\ and \\ Institute of Applied Problems of \\ Mathematics and Mechanics \\ Naukova Str. 3b \\ 79601, Lviv, Ukraine \\ mykytyuk_i@yahoo.com
}

\begin{abstract}
Let $G / K$ be an orbit of the adjoint representation of a compact connected Lie group $G, \sigma$ be an involutive automorphism of $G$ and $\widetilde{G}$ be the Lie group of fixed points of $\sigma$. We find a sufficient condition for the complete integrability of the geodesic flow of the Riemannian metric on $\widetilde{G} /(\widetilde{G} \cap K)$ which is induced by the bi-invariant Riemannian metric on $\widetilde{G}$. The integrals constructed here are real analytic functions, polynomial in momenta. It is checked that this sufficient condition holds when $G$ is the unitary group $\mathrm{U}(n)$ and $\sigma$ is its automorphism determined by the complex conjugation.
\end{abstract}

\section{Introduction}

Let $G / K$ be a homogeneous space of a compact Lie group $G$. We consider the problem of the complete integrability of the geodesic flow of the Riemannian metric on $G / K$ which is induced by a bi-invariant Riemannian metric on $G$. This problem was solved for some types of homogeneous manifolds including symmetric spaces, spherical spaces, Stiefel manifolds, flag manifolds, orbits of the adjoint actions, and others (see [Ma], [Mi], [GS], [My2], [MS], [BJ1], [BJ3], [MP]). Here we consider the new family of homogeneous manifolds, namely, the suborbits of orbits of the adjoint actions.

This paper is motivated by the paper [DGJ1], in which there were constructed integrable geodesic flows of $\widetilde{G}$-invariant metrics on the homogeneous space $\widetilde{G} / \widetilde{K}=$ $\mathrm{SO}(n) /\left(\mathrm{SO}\left(n_{1}\right) \times \cdots \times \mathrm{SO}\left(n_{p}\right)\right), n_{1}+\cdots+n_{p}=n\left(n_{i} \geqslant 1, p \geqslant 3\right)$. The method

DOI: $10.1007 / \mathrm{s} 00031-016-9373-\mathrm{x}$

* This research is partially supported by the Ministry of Economy and Competitiveness, Spain - CSIC, under Project MTM2011-22528.

Received May 16, 2014. Accepted February 22, 2016.

Published online March 28, 2016.

Corresponding Author: I. V. Mykytyuk, e-mail: mykytyuk_i@yahoo.com. 
of the proof in [DGJ1] is based on investigations of bi-Poisson structures on the Lie algebras $\mathfrak{u}(n)$ and $\mathfrak{s o}(n)$ associated with Lie algebra deformations. We consider the Lie-algebraic aspects of the integrability problem for such homogeneous spaces. Our approach is based on the following observation: the space $\widetilde{G} / \widetilde{K}$ is a $\widetilde{G}$-suborbit of the adjoint orbit $G / K=\mathrm{U}(n) /\left(\mathrm{U}\left(n_{1}\right) \times \cdots \times \mathrm{U}\left(n_{p}\right)\right)$ of the Lie algebra $\mathfrak{u}(n)$ of the unitary group, i.e., $\widetilde{G} / \widetilde{K}=\operatorname{Ad}(\widetilde{G})(a)$, where $a \in \mathfrak{u}(n)$, and $G / K=\operatorname{Ad}(G)(a)$. Moreover, $\widetilde{G}$ is the group of fixed points of the involutive automorphism $\sigma$ of $\mathrm{U}(n)$ induced by the complex conjugation. In other words, the space $\widetilde{G} / \widetilde{K}$ is uniquely determined by the pair $(G / K, \sigma)$, where $G / K=\operatorname{Ad}(G)(a)$ is an arbitrary adjoint orbit of the Lie group $G$ in its Lie algebra $\mathfrak{g}$ with $a \in\left(1-\sigma_{*}\right) \mathfrak{g}$, and $\sigma_{*}$ is the tangent automorphism of the Lie algebra $\mathfrak{g}$.

Let $G$ be an arbitrary compact connected Lie group with an involutive automorphism $\sigma: G \rightarrow G$ and let $\widetilde{G}$ be the set of fixed points of $\sigma$. In this article we investigate the integrability of the geodesic flow on the cotangent bundle $T^{*}(\widetilde{G} / \widetilde{K})$ defined by a $\widetilde{G}$-invariant metric on $\widetilde{G} / \widetilde{K}$ which is induced by a bi-invariant Riemannian metric on $\widetilde{G}$. As a homogeneous space $\widetilde{G} / \widetilde{K}$, we consider the homogeneous space associated with the adjoint orbit $G / K=\operatorname{Ad}(G)(a)$ of an arbitrary point $a \in\left(1-\sigma_{*}\right) \mathfrak{g}$, i.e., $\widetilde{K}=\widetilde{G} \cap K$. We find a sufficient purely algebraic condition for the integrability of this geodesic flow on the symplectic manifold $T^{*}(\widetilde{G} / \widetilde{K})$ (Theorem 10, Propositions 14 and 15). We prove that this sufficient condition holds when $G$ is the unitary group $\mathrm{U}(n)$ and $\sigma$ is its automorphism defined by the complex conjugation (Theorem 16). These results confirm the Mishchenko-Fomenko conjecture [MF2], which says that a noncommutative integrability implies a Liouville (complete) integrability by means of the integrals in the same functional class (the geodesic flow of a metric on $G / K$ induced by a bi-invariant metric on $G$ is integrable in a noncommutative sense by means of analytic, polynomial in momenta functions [BJ1]). Our approach is based on the fact that $\widetilde{G} / \widetilde{K} \subset G / K$ is a totally real (Lagrangian) submanifold of the homogeneous Kähler manifold (the compact orbit) $G / K$, and $T(\widetilde{G} / \widetilde{K}) \subset T(G / K)$ is a totally real submanifold of $T(G / K)$. However, to simplify calculations we reformulate this fact in some algebraic terms (not explicitly, because explicit reformulation would be very complicated from the point of view of calculations on $T(T(G / K))$ ). Also, matters are considerably simplified by using a very interesting observation of Bolsinov and Jovanović [BJ3] about the set of roots associated with the homogeneous space (orbit) $G / K$ and the results of Kostant [Ko] describing all maximal dimension orbits of semisimple complex Lie algebras.

One calls a Hamiltonian system on $T^{*} M$ (completely) integrable if it admits the maximal number of independent integrals in involution, i.e., $\operatorname{dim} M$ functions commuting with respect to the Poisson bracket on $T^{*} M$ whose differentials are independent in an open dense subset of $T^{*} M$. This set of integrals is a complete involutive subset of the algebra $C^{\infty}\left(T^{*} M\right)$. By Liouville's theorem the integral curves of an integrable Hamiltonian system under a certain additional compactness assumption are quasiperiodic (are orbits of a constant vector field on an invariant torus).

Let $A^{G}$ be the set of all $G$-invariant real analytic functions on the cotangent 
bundle $T^{*} M$ of $M=G / K$. This space is an algebra with respect to the canonical Poisson bracket on the symplectic manifold $T^{*} M$. The natural extension of the action of $G$ on $M$ to an action on the symplectic manifold $T^{*} M$ is Hamiltonian with the moment mapping $\mu^{\text {can }}: T^{*} M \rightarrow \mathfrak{g}^{*}$. The functions of the type $h \circ \mu^{\text {can }}, h: \mathfrak{g}^{*} \rightarrow \mathbb{R}$, are integrals for any $G$-invariant Hamiltonian flow on $T^{*} M$, in particular, for the geodesic flow corresponding to any $G$-invariant Riemannian metric on $M$. In general, a complete involutive subset of $\left\{h \circ \mu^{\text {can }}, h: \mathfrak{g}^{*} \rightarrow \mathbb{R}\right\}$ is not a complete involutive subset of the algebra $C^{\infty}\left(T^{*} M\right)$. However, for the compact Lie group $G$ the problem of constructing a complete involutive set of real analytic functions on $T^{*}(G / K)$ is reduced to the problem of finding a complete involutive set of real analytic functions from the set $A^{G}$ (see [My3, §2], [BJ1, Lem. 3], $[\mathrm{Pa}])$. This is true also for the group $\widetilde{G}$ and the corresponding algebra $A^{\widetilde{G}} \subset C^{\infty}\left(T^{*}(\widetilde{G} / \widetilde{K})\right)$.

The algebra of functions $A^{G}$ on $T^{*}(G / K)$, where, recall, $G / K$ is the adjoint orbit, contains some complete involutive subset $\mathcal{F}$ of $A^{G}$ ([MP, Thm. 3.10]; see also [BJ2], [BJ3]). The homogeneous space $\widetilde{G} / \widetilde{K}$, as we remarked above, is a submanifold of $G / K$ and therefore $T(\widetilde{G} / \widetilde{K})$ is a submanifold of $T(G / K)$. Moreover, $T(\widetilde{G} / \widetilde{K})$ is a symplectic submanifold of $T(G / K)$, where the symplectic structures on these spaces are defined via isomorphisms $T(\widetilde{G} / \widetilde{K}) \simeq T^{*}(\widetilde{G} / \widetilde{K})$ and $T(G / K) \simeq T^{*}(G / K)$ using a standard $G$-invariant metric on $G / K$ and its restriction to $\widetilde{G} / \widetilde{K}$ (see Proposition 4). The set $\widetilde{\mathcal{F}}=\left\{\left.f\right|_{T(\widetilde{G} / \widetilde{K})}, f \in \mathcal{F}\right\}$ of restrictions is an involutive subset of the algebra $A^{\widetilde{G}}$. This involutiveness of the functions from $\widetilde{\mathcal{F}}$ is a consequence of the fact that $G / \widetilde{G}$ is a symmetric space and follows easily from results published in [MF1], $[\mathrm{TF}]$. The following observation is crucial in our approach:

If the functions from the set $\mathcal{F}$ are independent at some point of the symplectic submanifold $T(\widetilde{G} / \widetilde{K}) \subset T(G / K)$, then the set $\widetilde{\mathcal{F}}$ is a complete involutive subset of the algebra $A^{\widetilde{G}}$.

Therefore we investigate some open dense subset $O$ of $T(G / K)$, where all functions from the set $\mathcal{F}$ are independent (in the paper [MP] only the existence of such a set was proved). We prove that $O \cap T(\widetilde{G} / \widetilde{K}) \neq \varnothing$ if $G$ is the unitary group $\mathrm{U}(n)$ and $\sigma$ is its automorphism determined by the complex conjugation (Theorem 16).

The present paper is also motivated by the following observation: in the above mentioned paper [DGJ1] the first part of the proof of Theorem 4 needs an additional argumentation (see more detailed comments in Remark 17). Nevertheless, the assertion of this theorem is true as follows from our result.

Note that the existence of complete commutative subalgebras of $A^{G}$ is an interesting and non-trivial problem. In the review paper [BJ4] this problem was formulated as Conjecture 6.1 in terms of the so-called integrable pairs of Lie groups $(G, K)$ (in the case of compact Lie groups). The present paper proves this conjecture for a series of important particular cases.

Acknowledgement. I thank the anonymous referees for a thorough reading of the manuscript and a comprehensive list of suggestions that helped me to improve the presentation. 


\section{Some definitions, conventions, and notations}

All objects in this paper are real analytic, $X$ stands for a connected manifold, and $\mathcal{E}(X)$ for the space of real analytic functions on $X$.

We will say that some functions from the set $\mathcal{E}(X)$ are independent if their differentials are independent at each point of some open dense subset in $X$. For any subset $\mathcal{F} \subset \mathcal{E}(X)$ denote by $\operatorname{ddim}_{x} \mathcal{F}$ the maximal number of independent functions from the set $\mathcal{F}$ at a point $x \in X$. Put $\operatorname{ddim} \mathcal{F} \stackrel{\text { def }}{=} \max _{x \in X} \operatorname{ddim}_{x} \mathcal{F}$.

Let $\eta$ be a Poisson bi-vector on $X$ and let $\mathcal{A} \subset \mathcal{E}(X)$ be a Poisson subalgebra of $(\mathcal{E}(X), \eta)$, i.e., $\mathcal{A}$ is a real vector space closed under the Poisson bracket $\{$,$\} :$ $\left(f_{1}, f_{2}\right) \mapsto \eta\left(d f_{1}, d f_{2}\right)$ on $X$. Put $(D \mathcal{A})_{x} \stackrel{\text { def }}{=}\left\{d f_{x}: f \in \mathcal{A}\right\} \subset T_{x}^{*} X$ for any $x \in X$. Let $B_{x}$ denote the restriction of $\eta_{x}$ to this subspace $(D \mathcal{A})_{x}$. We say that a subset $\mathcal{F} \subset \mathcal{A}$ is a complete involutive subset of the algebra $(\mathcal{A}, \eta)$ if at each point $x$ of some open dense subset in $X$ the subspace $V_{x} \stackrel{\text { def }}{=}\left\langle d f_{x}: f \in \mathcal{F}\right\rangle \subset(D \mathcal{A})_{x}$ is maximal isotropic with respect to the form $B_{x}$, i.e., $B_{x}\left(V_{x}, V_{x}\right)=0$ and $B_{x}\left(v, V_{x}\right)=0$ for $v \in(D \mathcal{A})_{x}$ implies $v \in V_{x}$. In particular, any two functions $f_{1}, f_{2} \in \mathcal{F}$ are in involution on $X$, i.e., $\left\{f_{1}, f_{2}\right\}=0$.

Definition 1. A pair $\left(\eta_{1}, \eta_{2}\right)$ of linearly independent bi-vector fields (bi-vectors for short) on a manifold $X$ is called Poisson if $\eta^{t} \stackrel{\text { def }}{=} t_{1} \eta_{1}+t_{2} \eta_{2}$ is a Poisson bivector for any $t=\left(t_{1}, t_{2}\right) \in \mathbb{R}^{2}$, i.e., each bi-vector $\eta^{t}$ determines on $X$ a Poisson structure with the Poisson bracket $\{,\}^{t}:\left(f_{1}, f_{2}\right) \mapsto \eta^{t}\left(d f_{1}, d f_{2}\right)$; the whole family of Poisson bi-vectors $\left\{\eta^{t}\right\}_{t \in \mathbb{R}^{2}}$ is called a bi-Poisson structure.

A bi-Poisson structure $\left\{\eta^{t}\right\}$ (we will often skip the parameter space) can be viewed as a two-dimensional vector space of Poisson bi-vectors, the Poisson pair $\left(\eta_{1}, \eta_{2}\right)$ as a basis in this space.

Suppose that a linear subspace $\mathcal{A} \subset \mathcal{E}(X)$ is a Poisson subalgebra of $\left(\mathcal{E}(X), \eta^{t}\right)$ for each $t \in \mathbb{R}^{2} \backslash\{0\}$. Let $B_{x}^{t}$ denote the restriction of $\eta_{x}^{t}$ to the subspace $(D \mathcal{A})_{x}$, $x \in X$.

Definition 2. We say that the pair $\left(\mathcal{A},\left\{\eta^{t}\right\}\right)$ is Kronecker at a point $x \in X$ for which $\operatorname{ddim}_{x} \mathcal{A}=\operatorname{ddim} \mathcal{A}$ if the linear space $\left\{B_{x}^{t}, t \in \mathbb{C}^{2}\right\}$ is two-dimensional and $\operatorname{rank}_{\mathbb{C}} B_{x}^{t}$ is constant with respect to $\left(t_{1}, t_{2}\right) \in \mathbb{C}^{2} \backslash\{0\}$. We regard $B_{x}^{t}, t \in \mathbb{C}^{2}$, as a complex bilinear form $t_{1} B_{x}^{(1,0)}+t_{2} B_{x}^{(0,1)}$ on the complexification $(D \mathcal{A})_{x}^{\mathbb{C}} \subset\left(T_{x}^{*} X\right)^{\mathbb{C}}$.

The definitions above are motivated by the following assertion of Bolsinov which is fundamental for our considerations.

Proposition 3. [Bo] Let $B_{1}$ and $B_{2}$ be two linearly independent skew-symmetric bilinear forms on a vector space $V$. Suppose that the kernel of each form $B^{t}=$ $t_{1} B_{1}+t_{2} B_{2}, t \in \mathbb{R}^{2}$, is non-trivial, i.e., $0<r \stackrel{\text { def }}{=} \min _{t \in \mathbb{R}^{2}} \operatorname{dim} \operatorname{ker} B^{t}$. Put $T=\{t \in$ $\left.\mathbb{R}^{2}: \operatorname{dim} \operatorname{ker} B^{t}=r\right\}$. Then

(1) the subspace $L \stackrel{\text { def }}{=} \sum_{t \in T} \operatorname{ker} B^{t}$ is isotropic with respect to any form $B^{t}$, $t \in \mathbb{R}^{2}$, i.e., $B^{t}(L, L)=0$;

(2) the space $L$ is maximal isotropic with respect to any form $B^{t}, t \in T$ if and only if $\operatorname{dim}_{\mathbb{C}} \operatorname{ker} B^{t}=r$ for all $t \in \mathbb{C}^{2} \backslash\{0\}$. 
Consider a connected Riemannian manifold $(M, \mathbf{g})$ and its connected Riemannian submanifold $(\widetilde{M}, \widetilde{\mathbf{g}})$, where $\widetilde{\mathbf{g}}=\left.\mathbf{g}\right|_{\widetilde{M}}$. The cotangent bundles $T^{*} M$ and $T^{*} \widetilde{M}$ are symplectic manifolds with the canonical symplectic structures $\Omega$ and $\widetilde{\Omega}$ respectively. Using the metric $\mathbf{g}$ (resp. $\widetilde{\mathbf{g}}$ ) we can identify $T^{*} M$ with $T M$ (resp. $T^{*} \widetilde{M}$ with $T \widetilde{M}$ ), denoting by $\varphi: T M \rightarrow T^{*} M$ (resp. $\widetilde{\varphi}: T \widetilde{M} \rightarrow T^{*} \widetilde{M}$ ) the corresponding diffeomorphism. Let $p: T M \rightarrow M(\operatorname{resp} . \widetilde{p}: T \widetilde{M} \rightarrow \widetilde{M})$ be the natural projection and let $\theta$ (resp. $\widetilde{\theta}$ ) be the canonical 1-form on $T^{*} M\left(\operatorname{resp}\right.$. on $\left.T^{*} \widetilde{M}\right)$.

Proposition 4. The symplectic manifold $\left(T \widetilde{M}, \widetilde{\varphi}^{*} \widetilde{\Omega}\right)$ is a symplectic submanifold of $\left(T M, \varphi^{*} \Omega\right)$, i.e., $\widetilde{\varphi}^{*} \widetilde{\Omega}=\left.\varphi^{*} \Omega\right|_{T \widetilde{M}}$. Moreover, $\widetilde{\varphi}^{*} \widetilde{\theta}=\left.\varphi^{*} \theta\right|_{T \widetilde{M}}$.

Proof. By the definition, $\theta_{x^{\prime}}\left(Z^{\prime}\right)=x^{\prime}\left(\pi_{* x^{\prime}} Z^{\prime}\right)$, where $\pi: T^{*} M \rightarrow M$ is the natural projection, $x^{\prime} \in T_{q}^{*} M, q=\pi\left(x^{\prime}\right), Z^{\prime} \in T_{x^{\prime}}\left(T^{*} M\right)$.

If $x, y \in T_{q} M$, then $\varphi(x) \in T_{q}^{*} M$ and, by the definition, $\varphi(x)(y)=\mathbf{g}_{q}(x, y)$. Putting $x^{\prime}=\varphi(x) \in T_{q}^{*} M$ (to simplify the notation) and taking into account that $\pi \circ \varphi=p$ we obtain that for any $Z \in T_{x} T M$

$$
\begin{aligned}
\left(\varphi^{*} \theta\right)_{x}(Z) & \stackrel{\text { def }}{=} \theta_{\varphi(x)}\left(\varphi_{* x} Z\right) \stackrel{\text { def }}{=} x^{\prime}\left(\pi_{* x^{\prime}}\left(\varphi_{* x} Z\right)\right) \\
& =x^{\prime}\left((\pi \circ \varphi)_{* x} Z\right)=x^{\prime}\left(p_{* x} Z\right)=\mathbf{g}_{q}\left(x, p_{* x} Z\right)
\end{aligned}
$$

Similarly, we obtain that

$$
\left(\widetilde{\varphi}^{*} \widetilde{\theta}\right)_{\widetilde{x}}(\widetilde{Z})=\widetilde{\mathbf{g}}_{\widetilde{q}}\left(\widetilde{x}, \widetilde{p}_{* \widetilde{x}} \widetilde{Z}\right) \quad \text { for any } \quad \widetilde{q} \in \widetilde{M}, \widetilde{x} \in T_{\widetilde{q}} \widetilde{M}, \widetilde{Z} \in T_{\widetilde{x}} T \widetilde{M}
$$

In other words, $\widetilde{\varphi}^{*} \widetilde{\theta}=\left.\varphi^{*} \theta\right|_{T \widetilde{M}}$, because $\left.p\right|_{T \widetilde{M}}=\widetilde{p}$ and $\left.\mathbf{g}\right|_{\widetilde{M}}=\widetilde{\mathbf{g}}$. Now to complete the proof it is sufficient to note that $\Omega=d \theta$ and $\widetilde{\Omega}=d \widetilde{\theta}$.

\section{The integrability of geodesic flows}

In this section, for any compact Lie algebra $\mathfrak{a}$ by $\mathfrak{z}(\mathfrak{a})$ we will denote its center and by $\mathfrak{a}_{s}$ its maximal semisimple ideal, i.e., $\mathfrak{a}=\mathfrak{z}(\mathfrak{a}) \oplus \mathfrak{a}_{s}$; for any real vector space or any Lie algebra $\mathfrak{a}$ by $\mathfrak{a}^{\mathbb{C}}$ we will denote its complexification.

\subsection{Commutator on $A_{\mathfrak{m}}^{K}$ induced by canonical Poisson structure on $T^{*}(G / K)$}

Let $M=G / K$ be a homogeneous space of a compact connected Lie group $G$ with the Lie algebra $\mathfrak{g}$. There exists a faithful representation $\chi$ of $\mathfrak{g}$ such that its associated bilinear form $\Phi_{\chi}$ is negative-definite on $\mathfrak{g}$ (if $\mathfrak{g}$ is semi-simple we can take the Killing form associated with the adjoint representation of $\mathfrak{g})$. Let $\mathfrak{m}=\mathfrak{k}^{\perp}$ be the orthogonal complement to $\mathfrak{k}$ with respect to $\Phi_{\chi}$. Then

$$
\mathfrak{g}=\mathfrak{m} \oplus \mathfrak{k}, \quad[\mathfrak{k}, \mathfrak{m}] \subset \mathfrak{m} .
$$

The form $\langle\rangle=,-\Phi_{\chi}$ determines a $G$-invariant metric on $G / K$. This metric identifies the cotangent bundle $T^{*}(G / K)$ and the tangent bundle $T(G / K)$. Thus we can also talk on the canonical 2 -form $\Omega$ on the manifold $T(G / K)$. The symplectic form $\Omega$ is $G$-invariant with respect to the natural action of $G$ on $T(G / K)$ (extension of the action of $G$ on $G / K$ ). 
We can identify the tangent space $T_{o}(G / K)$ at the point $o=p(e)$ with the space $\mathfrak{m}$ by means of the canonical projection $p: G \rightarrow G / K$. Let $A^{G}$ (resp. $A_{\mathfrak{m}}^{K}$ ) be the set of all $G$-invariant (resp. $\operatorname{Ad}(K)$-invariant) functions on $T(G / K)$ (resp. on $\mathfrak{m})$. There is a one-to-one correspondence between $G$-orbits in $T(G / K)$ and $\operatorname{Ad}(K)$-orbits in $\mathfrak{m}$. Thus we can naturally identify the spaces of functions $A^{G}$ and $A_{\mathfrak{m}}^{K}$. For any smooth function $f$ on $\mathfrak{m}$ write $\operatorname{grad}_{\mathfrak{m}} f$ for the vector field on $\mathfrak{m}$ such that

$$
d f_{x}(y)=\left\langle\operatorname{grad}_{\mathfrak{m}} f(x), y\right\rangle \quad \text { for all } y \in \mathfrak{m} .
$$

The Poisson bracket of two functions $f_{1}, f_{2}$ from the set $A_{\mathfrak{m}}^{K}=A^{G}$ with respect to the canonical Poisson structure $\eta^{\text {can }}$ (determined by the canonical 2-form $\Omega$ ) has the form [MP, Lem. 3.1] :

$$
\left\{f_{1}, f_{2}\right\}^{\operatorname{can}}(x)=-\left\langle x,\left[\operatorname{grad}_{\mathfrak{m}} f_{1}(x), \operatorname{grad}_{\mathfrak{m}} f_{2}(x)\right]\right\rangle, \quad x \in \mathfrak{m} .
$$

Now, let us consider an important (for our considerations) subspace of $\mathfrak{m}$. For any $x \in \mathfrak{m}$ define the subspace $\mathfrak{m}(x) \subset \mathfrak{m}$ putting

$$
\mathfrak{m}(x) \stackrel{\text { def }}{=}\{y \in \mathfrak{m}:[x, y] \in \mathfrak{m}\}=\{y \in \mathfrak{m}:\langle y, \operatorname{ad} x(\mathfrak{k})\rangle=0\}
$$

in particular,

$$
\operatorname{ad} x(\mathfrak{m}(x)) \subset \mathfrak{m} \quad \text { and } \quad \mathfrak{m}(x) \oplus \operatorname{ad} x(\mathfrak{k})=\mathfrak{m} .
$$

For any element $x \in \mathfrak{g}$ denote by $\mathfrak{g}^{x}$ its centralizer in $\mathfrak{g}$, i.e., the set of all $z \in \mathfrak{g}$ satisfying $[x, z]=0$. Put $\mathfrak{k}^{x}=\mathfrak{g}^{x} \cap \mathfrak{k}$. Consider in $\mathfrak{m}$ a nonempty Zariski open subset:

$$
R(\mathfrak{m})=\left\{x \in \mathfrak{m}: \operatorname{dim} \mathfrak{g}^{x}=q(\mathfrak{m}), \operatorname{dim} \mathfrak{k}^{x}=p(\mathfrak{m})\right\}
$$

where $q(\mathfrak{m})$ (resp. $p(\mathfrak{m}))$ is the minimum of dimensions of the spaces $\mathfrak{g}^{y}$ (resp. $\left.\mathfrak{k}^{y}\right)$ over all $y \in \mathfrak{m}$.

Let $(\cdot)_{\mathfrak{m}}$ be the projection of $\mathfrak{g}$ into $\mathfrak{m}$ along $\mathfrak{k}$. For each $x \in R(\mathfrak{m})$ the spaces $\mathfrak{m}(x)$ and $\left(\mathfrak{g}^{x}\right)_{\mathfrak{m}} \subset \mathfrak{m}(x)$ have the same dimensions:

$$
\operatorname{dim} \mathfrak{m}(x)=\operatorname{dim} \mathfrak{m}-\operatorname{dim} \operatorname{ad} x(\mathfrak{k}) \quad \text { and } \quad \operatorname{dim}\left(\mathfrak{g}^{x}\right)_{\mathfrak{m}}=\operatorname{dim} \mathfrak{g}^{x}-\operatorname{dim} \mathfrak{k}^{x}
$$

Moreover, for each $x \in R(\mathfrak{m})$ the maximal semi-simple ideal $\left(\mathfrak{g}^{x}\right)_{s}=\left[\mathfrak{g}^{x}, \mathfrak{g}^{x}\right]$ of $\mathfrak{g}^{x}$ is contained in the algebra $\mathfrak{k}^{x}$, i.e.,

$$
\left[\mathfrak{g}^{x}, \mathfrak{g}^{x}\right]=\left[\mathfrak{k}^{x}, \mathfrak{k}^{x}\right], \quad \text { if } x \in R(\mathfrak{m})
$$

(see [My3, Prop.10] or [Mi]). Therefore, $\operatorname{dim}\left(\mathfrak{g}^{x} / \mathfrak{k}^{x}\right)=\operatorname{rank} \mathfrak{g}-\operatorname{rank} \mathfrak{k}^{x}$, i.e.,

$$
\operatorname{dim} \mathfrak{g}^{x}=\operatorname{rank} \mathfrak{g}+\left(\operatorname{dim} \mathfrak{k}^{x}-\operatorname{rank} \mathfrak{k}^{x}\right) \quad \text { if } x \in R(\mathfrak{m}) .
$$

It is clear that $\operatorname{grad}_{\mathfrak{m}} f(x) \in \mathfrak{m}(x)$ for any $f \in A_{\mathfrak{m}}^{K}$. Moreover, since the Lie group $K$ is compact, for each $x$ from some nonempty Zariski open subset of $\mathfrak{m}$ the space $\mathfrak{m}(x)$ is generated by the vectors $\operatorname{grad}_{\mathfrak{m}} f(x), f \in A_{\mathfrak{m}}^{K}$. For any $x \in R(\mathfrak{m})$ the 
kernel of the 2 -form $B_{x}^{0}: \mathfrak{m}(x) \times \mathfrak{m}(x) \rightarrow \mathbb{R},\left(y_{1}, y_{2}\right) \mapsto-\left\langle x,\left[y_{1}, y_{2}\right]\right\rangle$, associated with the Poisson structure $\eta^{\text {can }}$, is the space $\left(\mathfrak{g}^{x}\right)_{\mathfrak{m}}$ :

$$
\begin{aligned}
\{y \in \mathfrak{m}(x):\langle x,[y, \mathfrak{m}(x)]\rangle=0\} & =\{y \in \mathfrak{m}(x):\langle[x, y], \mathfrak{m}(x)\rangle=0\} \\
& =\{y \in \mathfrak{m}(x):[x, y] \in \operatorname{ad} x(\mathfrak{k})\} \\
& =\left(\mathfrak{g}^{x}\right)_{\mathfrak{m}} \cap \mathfrak{m}(x)=\left(\mathfrak{g}^{x}\right)_{\mathfrak{m}}
\end{aligned}
$$

Hence the number $(r(\mathfrak{m})+\operatorname{dim} \mathfrak{m}(x)) / 2$, where

$$
r(\mathfrak{m})=\operatorname{dim}\left(\mathfrak{g}^{x}\right)_{\mathfrak{m}}=\operatorname{dim} \mathfrak{g}^{x}-\operatorname{dim} \mathfrak{k}^{x}=q(\mathfrak{m})-p(\mathfrak{m}), \quad x \in R(\mathfrak{m}),
$$

is the maximal number of functions in involution from the set $A_{\mathfrak{m}}^{K}$ functionally independent at the point $x$.

For an arbitrary element $x \in R(\mathfrak{m})$, we have [My3, Prop.9]

$$
\left[\mathfrak{m}(x), \mathfrak{k}^{x}\right]=0 .
$$

The compact Lie algebra $\mathfrak{k}$ is the direct sum $\mathfrak{k}=\mathfrak{z}(\mathfrak{k}) \oplus \mathfrak{k}_{s}$ of the center and of the maximal semisimple ideal. The center $\mathfrak{z}(\mathfrak{k})$ of $\mathfrak{k}$ will be denoted simply by $\mathfrak{z}$ for short. Then we have the following orthogonal splittings with respect to the invariant form $\langle\cdot, \cdot\rangle: \mathfrak{k}=\mathfrak{z} \oplus \mathfrak{k}_{s}, \mathfrak{g}=(\mathfrak{m} \oplus \mathfrak{z}) \oplus \mathfrak{k}_{s}$.

Consider the set $R(\mathfrak{m} \oplus \mathfrak{z})$ determined by (4) for the pair $\left(\mathfrak{g}, \mathfrak{k}_{s}\right)$. Then for any $x+z \in \mathfrak{m} \oplus \mathfrak{z}$ such that $x \in R(\mathfrak{m}), z \in \mathfrak{z}$ and $x+z \in R(\mathfrak{m} \oplus \mathfrak{z})$, we have $\mathfrak{k}_{s}^{x+z}=\mathfrak{k}_{s}^{x}$ because $[\mathfrak{z}, \mathfrak{k}]=0$. However, $\mathfrak{k}_{s}$ contains the maximal semi-simple ideal of the compact Lie algebra $\mathfrak{k}^{x}$, and therefore $\left(\operatorname{dim} \mathfrak{k}^{x}-\operatorname{rank} \mathfrak{k}^{x}\right)=\left(\operatorname{dim} \mathfrak{k}_{s}^{x}-\operatorname{rank} \mathfrak{k}_{s}^{x}\right)$. Thus by (6)

$$
\begin{aligned}
q(\mathfrak{m}) & =\operatorname{dim} \mathfrak{g}^{x}=\operatorname{rank} \mathfrak{g}+\left(\operatorname{dim} \mathfrak{k}^{x}-\operatorname{rank} \mathfrak{k}^{x}\right)=\operatorname{rank} \mathfrak{g}+\left(\operatorname{dim} \mathfrak{k}_{s}^{x}-\operatorname{rank} \mathfrak{k}_{s}^{x}\right) \\
& =\operatorname{rank} \mathfrak{g}+\left(\operatorname{dim} \mathfrak{k}_{s}^{x+z}-\operatorname{rank} \mathfrak{k}_{s}^{x+z}\right)=\operatorname{dim} \mathfrak{g}^{x+z}=q(\mathfrak{m} \oplus \mathfrak{z}) .
\end{aligned}
$$

The following proposition will be often used in Subsections 2.2 and 2.3.

Proposition 5. Suppose that $\mathfrak{k}=\mathfrak{g}^{a}$ for some $a \in \mathfrak{g}$ and $x_{0} \in R(\mathfrak{m})$. Let $\mathfrak{g}_{0}$ and $\mathfrak{k}_{0}$ be the centralizers of the algebra $\mathfrak{k}^{x_{0}}$ in $\mathfrak{g}$ and $\mathfrak{k}$ respectively. Let $\mathfrak{m}_{0}=\mathfrak{g}_{0} \cap \mathfrak{m}$. Then

(1) for any $x \in \mathfrak{m}_{0} \cap R(\mathfrak{m})$ (this set contains $x_{0}$ ) we have $\mathfrak{m}_{0}(x)=\mathfrak{m}(x)$;

(2) for any $x \in \mathfrak{m}_{0} \cap R(\mathfrak{m})$ we have $\mathfrak{k}^{x}=\mathfrak{k}^{x_{0}}$ and $x \in R\left(\mathfrak{m}_{0}\right)$;

(3) $a \in \mathfrak{g}_{0}, \mathfrak{g}^{x_{0}}=\mathfrak{g}_{0}^{x_{0}} \oplus\left(\mathfrak{k}^{x_{0}}\right)_{s}$ and the direct sum $\mathfrak{g}_{0} \oplus\left(\mathfrak{k}^{x_{0}}\right)_{s}$ is a subalgebra of $\mathfrak{g}$ of maximal rank;

(4) each element $x$ of the set $R\left(\mathfrak{m}_{0}\right)$ is a regular element of the Lie algebra $\mathfrak{g}_{0}$ and its centralizer $\mathfrak{k}_{0}^{x}$ in $\mathfrak{k}_{0}$ is the center $\mathfrak{z}\left(\mathfrak{g}_{0}\right)$ of $\mathfrak{g}_{0}$;

(5) $r(\mathfrak{m})=r\left(\mathfrak{m}_{0}\right)=\operatorname{rank} \mathfrak{g}_{0}-\operatorname{dim} \mathfrak{z}\left(\mathfrak{g}_{0}\right)$.

Proof. Items (1) and (2) follow immediately from Proposition 2.3 [MP]. It is clear that $a \in \mathfrak{g}_{0}$ because $a \in \mathfrak{z}(\mathfrak{k})$. By $(5),\left(\mathfrak{g}^{x_{0}}\right)_{s}=\left(\mathfrak{k}^{x_{0}}\right)_{s} \subset \mathfrak{k}$, i.e., $\mathfrak{g}^{x_{0}}=$ $\mathfrak{z}\left(\mathfrak{g}^{x_{0}}\right) \oplus\left(\mathfrak{k}^{x_{0}}\right)_{s}$ and $\mathfrak{k}^{x_{0}}=\mathfrak{z}\left(\mathfrak{k}^{x_{0}}\right) \oplus\left(\mathfrak{k}^{x_{0}}\right)_{s}$. Since by definition $\mathfrak{k}^{x_{0}} \subset \mathfrak{g}^{x_{0}}$, we obtain that $\left[\mathfrak{z}\left(\mathfrak{g}^{x_{0}}\right), \mathfrak{k}^{x_{0}}\right]=0$, i.e., $\mathfrak{z}\left(\mathfrak{g}^{x_{0}}\right) \subset \mathfrak{g}_{0}$, and consequently, $\mathfrak{g}_{0}^{x_{0}} \stackrel{\text { def }}{=} \mathfrak{g}^{x_{0}} \cap \mathfrak{g}_{0}=\mathfrak{z}\left(\mathfrak{g}^{x_{0}}\right)$. 
In other words, $x_{0}$ is a regular element of the Lie algebra $\mathfrak{g}_{0}$ and $\mathfrak{g}^{x_{0}}=\mathfrak{g}_{0}^{x_{0}} \oplus\left(\mathfrak{k}^{x_{0}}\right)_{s}$. Since the Lie algebra $\left(\mathfrak{k}^{x_{0}}\right)_{s}$ is semisimple, $\mathfrak{g}_{0} \cap\left(\mathfrak{k}^{x_{0}}\right)_{s}=0$. The subalgebra $\mathfrak{g}_{0} \oplus$ $\left(\mathfrak{k}^{x_{0}}\right)_{s} \subset \mathfrak{g}$ is a subalgebra of maximal rank because it contains the centralizer $\mathfrak{g}^{x_{0}}$.

Each element $x$ of the set $R\left(\mathfrak{m}_{0}\right)$ is also regular in $\mathfrak{g}_{0}$ because $x_{0} \in R\left(\mathfrak{m}_{0}\right)$ by (2) and by definition of the set $R\left(\mathfrak{m}_{0}\right)$ (see (4)), $\operatorname{dim} \mathfrak{g}_{0}^{x}=\operatorname{dim} \mathfrak{g}_{0}^{x_{0}}\left(=\operatorname{rank} \mathfrak{g}_{0}\right)$.

By the definition, $\mathfrak{z}\left(\mathfrak{k}^{x_{0}}\right)=\mathfrak{k}^{x_{0}} \cap \mathfrak{g}_{0}$ and $\mathfrak{z}\left(\mathfrak{k}^{x_{0}}\right) \subset \mathfrak{z}\left(\mathfrak{g}_{0}\right)$. Since $\mathfrak{k}_{0}^{x_{0}} \stackrel{\text { def }}{=} \mathfrak{k}^{x_{0}} \cap$ $\mathfrak{g}_{0}$, we obtain that $\mathfrak{k}_{0}^{x_{0}}=\mathfrak{z}\left(\mathfrak{k}^{x_{0}}\right)$ and $\mathfrak{k}_{0}^{x_{0}} \subset \mathfrak{z}\left(\mathfrak{g}_{0}\right)$. But $a, x_{0} \in \mathfrak{g}_{0}$. Hence these elements commute with the center $\mathfrak{z}\left(\mathfrak{g}_{0}\right)$ of $\mathfrak{g}_{0}$ and, consequently, $\mathfrak{z}\left(\mathfrak{g}_{0}\right) \subset \mathfrak{g}^{a}=\mathfrak{k}$ and $\mathfrak{z}\left(\mathfrak{g}_{0}\right) \subset \mathfrak{g}^{x_{0}}$. In other words, $\mathfrak{z}\left(\mathfrak{g}_{0}\right) \subset \mathfrak{k} \cap \mathfrak{g}^{x_{0}} \cap \mathfrak{g}_{0} \stackrel{\text { def }}{=} \mathfrak{k}_{0}^{x_{0}}$, i.e., $\mathfrak{k}_{0}^{x_{0}}=\mathfrak{z}\left(\mathfrak{g}_{0}\right)$. Since $\operatorname{dim} \mathfrak{k}_{0}^{x_{0}}=\operatorname{dim} \mathfrak{k}_{0}^{x}$ for $x \in R\left(\mathfrak{m}_{0}\right)$ and $\mathfrak{z}\left(\mathfrak{g}_{0}\right) \subset\left(\mathfrak{g}_{0}^{x} \cap \mathfrak{k}\right) \stackrel{\text { def }}{=} \mathfrak{k}_{0}^{x}$, item (4) is proved. To complete the proof of the proposition it is sufficient to remark that $x_{0} \in R(\mathfrak{m}) \cap R\left(\mathfrak{m}_{0}\right)$,

$$
r(\mathfrak{m})=\operatorname{dim} \mathfrak{g}^{x_{0}}-\operatorname{dim} \mathfrak{k}^{x_{0}}=\operatorname{dim} \mathfrak{z}\left(\mathfrak{g}^{x_{0}}\right)-\operatorname{dim} \mathfrak{z}\left(\mathfrak{k}^{x_{0}}\right)=\operatorname{dim} \mathfrak{g}_{0}^{x_{0}}-\operatorname{dim} \mathfrak{k}_{0}^{x_{0}}=r\left(\mathfrak{m}_{0}\right)
$$

and $x_{0}$ is a regular element of $\mathfrak{g}_{0}$, i.e., $\operatorname{dim} \mathfrak{g}_{0}^{x_{0}}=\operatorname{rank} \mathfrak{g}_{0}$.

\subsection{The bi-Poisson structure $\left\{\eta^{t}(\mathfrak{g}, a)\right\}$ : exact formulas and involutive sets of functions}

Consider the adjoint action Ad of $G$ on the Lie algebra $\mathfrak{g}$. Suppose now in addition that the Lie subgroup $K$ is an isotropy group of some element $a \in \mathfrak{g}$, i.e., $K=$ $\{g \in G: \operatorname{Ad} g(a)=a\}$ and $\mathfrak{k}=\mathfrak{g}^{a}$. Moreover, by invariance of the form $\langle\cdot, \cdot\rangle$, $\mathfrak{k}=\mathfrak{z}(\mathfrak{k}) \oplus \mathfrak{k}_{s}, a \in \mathfrak{z}(\mathfrak{k})$, and $\operatorname{ad} a(\mathfrak{m}) \subset \mathfrak{m}$.

Using the invariant form $\langle\cdot, \cdot\rangle$ on the Lie algebra $\mathfrak{g}$, we identify the dual space $\mathfrak{g}^{*}$ and $\mathfrak{g}$. So the orbit $\mathcal{O}=G / K$ is a symplectic manifold with the Kirillov-KostantSouriau symplectic structure $\omega_{\mathcal{O}}$. By the definition, the form $\omega_{\mathcal{O}}$ is $G$-invariant and at the point $a \in \mathcal{O}$ we have

$$
\omega_{\mathcal{O}}(a)\left(\left[a, \xi_{1}\right],\left[a, \xi_{2}\right]\right)=-\left\langle a,\left[\xi_{1}, \xi_{2}\right]\right\rangle, \quad \forall \xi_{1}, \xi_{2} \in \mathfrak{g},
$$

where we consider the vectors $\left[a, \xi_{1}\right],\left[a, \xi_{2}\right] \in T_{a} \mathfrak{g}=\mathfrak{g}$ as tangent vectors to the orbit $\mathcal{O} \subset \mathfrak{g}$ at the point $a \in \mathcal{O}$. Let $\tau: T \mathcal{O} \rightarrow \mathcal{O}$ be the natural projection. Using the closed 2-form $\tau^{*} \omega_{\mathcal{O}}$ on $T \mathcal{O}$ (the lift of $\omega_{\mathcal{O}}$ ) we construct a bi-Poisson structure on $T \mathcal{O}$.

Consider on $T \mathcal{O}$ two symplectic forms: $\omega_{1}=\Omega$ and $\omega_{2}=\Omega+\tau^{*} \omega_{\mathcal{O}}$. Write $\eta_{1}=\omega_{1}^{-1}, \eta_{2}=\omega_{2}^{-1}$ for the standard Poisson bi-vectors associated with these forms, i.e., $\eta_{i}\left(d f_{1}, d f_{2}\right)=-\omega_{i}\left(\xi_{f_{1}}, \xi_{f_{2}}\right)$, where $\xi_{f_{j}}$, are the Hamiltonian vector fields of the functions $f_{j}\left(d f_{j}=-\omega_{i}\left(\xi_{f_{j}}, \cdot\right)\right), i, j=1,2$. Then the family $\left\{\eta^{t}(\mathfrak{g}, a)=\eta^{t}=\right.$ $\left.t_{1} \eta_{1}+t_{2} \eta_{2}\right\}, t_{1}, t_{2} \in \mathbb{R}$, is a bi-Poisson structure on $T \mathcal{O}$ [MP, Prop.1.6]. Putting $t_{2}=\lambda, t_{1}=1-\lambda, \lambda \in \mathbb{R}$ or $t_{1}=-1, t_{2}=1$, we exclude consideration of proportional bi-vectors. The corresponding bi-vectors are denoted by $\eta^{\lambda}, \lambda \in \mathbb{R}$, and $\eta^{\mathrm{si}}$ (the singular bi-vector). The Poisson bracket of two functions $f_{1}, f_{2}$ from the set $A_{\mathfrak{m}}^{K}=A^{G} \subset \mathcal{E}(T \mathcal{O})$ with respect to the Poisson structure $\eta^{\lambda}, \lambda \in \mathbb{R}$ or $\eta^{\text {si }}$ has the form [MP, Lem. 3.1]:

$$
\begin{aligned}
& \left\{f_{1}, f_{2}\right\}^{\lambda}(x)=-\left\langle x+\lambda a,\left[\operatorname{grad}_{\mathfrak{m}} f_{1}(x), \operatorname{grad}_{\mathfrak{m}} f_{2}(x)\right]\right\rangle, \\
& \left\{f_{1}, f_{2}\right\}^{\mathrm{si}}(x)=-\left\langle a,\left[\operatorname{grad}_{\mathfrak{m}} f_{1}(x), \operatorname{grad}_{\mathfrak{m}} f_{2}(x)\right]\right\rangle
\end{aligned}
$$


Notice that the structure $\eta^{0}(\lambda=0)$ is the canonical Poisson structure (see $\left.(2)\right)$. Since the bi-Poisson structure $\left\{\eta^{t}\right\}$ is $G$-invariant it is sufficient to investigate points in $R(\mathfrak{m}) \subset \mathfrak{m}=T_{o}(G / K)$, where the pair $\left(A_{\mathfrak{m}}^{K}, \eta^{t}\right)$ is Kronecker.

The bi-Poisson structure $\left\{\eta^{t}\right\}$ determines at a point $x \in \mathfrak{m}=T_{o}(G / K)$ the bilinear forms $B_{x}^{t}:\left(D A^{G}\right)_{x} \times\left(D A^{G}\right)_{x} \rightarrow \mathbb{R}$, where, recall, $B_{x}^{t}$ is the restriction $\left.\eta^{t}\right|_{\left(D A^{G}\right)_{x}}$. Let $x$ be an element of $R(\mathfrak{m}) \subset T_{o}(G / K)$. Since we identified the spaces $A^{G}$ and $A_{\mathfrak{m}}^{K}, B_{x}^{t}$ determines the following complex-valued bilinear forms (which we denote also by $B_{x}^{t}, B_{x}^{\lambda}$ and $B_{x}^{\text {si }}$ for short) on $\mathfrak{m}(x) \times \mathfrak{m}(x)[\mathrm{MP},(3.11)]$ :

$$
\begin{aligned}
& B_{x}^{t}:\left(y_{1}, y_{2}\right) \mapsto-\left\langle\left(t_{1}+t_{2}\right) x+t_{2} a,\left[y_{1}, y_{2}\right]\right\rangle, t_{1}, t_{2} \in \mathbb{C}, \\
& B_{x}^{\lambda}:\left(y_{1}, y_{2}\right) \mapsto-\left\langle x+\lambda a,\left[y_{1}, y_{2}\right]\right\rangle, \quad \lambda \in \mathbb{C}, \\
& B_{x}^{\mathrm{si}}:\left(y_{1}, y_{2}\right) \mapsto-\left\langle a,\left[y_{1}, y_{2}\right]\right\rangle .
\end{aligned}
$$

Let $\mathfrak{m}^{\mathbb{C}}(x)$ be the complexification of the space $\mathfrak{m}(x), x \in R(\mathfrak{m})$. It is easy to see that the kernel of the form $B_{x}^{\lambda}$ in $\mathfrak{m}^{\mathbb{C}}(x)$ is the subspace of $\mathfrak{m}^{\mathbb{C}}(x)$ given by

$$
\begin{aligned}
\operatorname{ker} B_{x}^{\lambda} & =\left\{y \in \mathfrak{m}^{\mathbb{C}}(x):[x+\lambda a, y] \in \operatorname{ad} x\left(\mathfrak{k}^{\mathbb{C}}\right)\right\} \\
& =\left\{y \in \mathfrak{m}^{\mathbb{C}}(x):[x+\lambda a, y] \in \operatorname{ad}(x+\lambda a)\left(\mathfrak{k}^{\mathbb{C}}\right)\right\}
\end{aligned}
$$

because $\operatorname{ad} x\left(\mathfrak{k}^{\mathbb{C}}\right)=\left(\mathfrak{m}^{\mathbb{C}}(x)\right)^{\perp}$ in $\mathfrak{m}^{\mathbb{C}}$ and $\left[a, \mathfrak{k}^{\mathbb{C}}\right]=0$. Thus

$$
\operatorname{ker} B_{x}^{\lambda}=\left(\left(\mathfrak{g}^{\mathbb{C}}\right)^{x+\lambda a}\right)_{\mathfrak{m} \mathbb{C}} \cap \mathfrak{m}^{\mathbb{C}}(x),
$$

where $(\cdot)_{\mathfrak{m}^{\mathbb{C}}}$ denotes the projection onto $\mathfrak{m}^{\mathbb{C}}$ along $\mathfrak{k}^{\mathbb{C}}$. However, $\left(\left(\mathfrak{g}^{\mathbb{C}}\right)^{x+\lambda a}\right)_{\mathfrak{m}^{\mathbb{C}}} \subset$ $\mathfrak{m}^{\mathbb{C}}(x)$ because $\left[a, \mathfrak{m}^{\mathbb{C}}\right] \subset \mathfrak{m}^{\mathbb{C}},\left[a, \mathfrak{k}^{\mathbb{C}}\right]=0$, ad $x\left(\mathfrak{k}^{\mathbb{C}}\right) \subset \mathfrak{m}^{\mathbb{C}}$ and by $(3) y \in \mathfrak{m}^{\mathbb{C}}$ is an element of $\mathfrak{m}^{\mathbb{C}}(x)$ if and only if $[x, y] \in \mathfrak{m}^{\mathbb{C}}$. Thus,

$$
\operatorname{ker} B_{x}^{\lambda}=\left(\left(\mathfrak{g}^{\mathbb{C}}\right)^{x+\lambda a}\right)_{\mathfrak{m}^{\mathbb{C}}}, \lambda \in \mathbb{C} .
$$

In particular, for $\lambda=0$ (for the canonical Poisson structure on $T(G / K)$ ),

$$
\operatorname{ker} B_{x}^{0}=\left(\left(\mathfrak{g}^{\mathbb{C}}\right)^{x}\right)_{\mathfrak{m}^{\mathbb{C}}}=\left(\left(\mathfrak{g}^{x}\right)_{\mathfrak{m}}\right)^{\mathbb{C}} .
$$

Since $x \in R(\mathfrak{m})$, the (real) dimension of the space $\left(\mathfrak{g}^{x}\right)_{\mathfrak{m}}$ is equal to the constant $r(\mathfrak{m})=q(\mathfrak{m})-p(\mathfrak{m})$. Therefore a maximal isotropic subspace of the space $\mathfrak{m}(x)$ with respect to the form $B_{x}^{0}$ is of dimension $(r(\mathfrak{m})+\operatorname{dim} \mathfrak{m}(x)) / 2$. It is clear that

$$
\operatorname{ker} B_{x}^{\mathrm{si}}=\left\{y \in \mathfrak{m}^{\mathbb{C}}(x):[a, y] \in \operatorname{ad} x\left(\mathfrak{k}^{\mathbb{C}}\right)\right\}=\mathfrak{m}^{\mathbb{C}}(x) \cap\left(\operatorname{ad}_{a}^{-1} \operatorname{ad} x\left(\mathfrak{k}^{\mathbb{C}}\right)\right),
$$

where $\operatorname{ad}_{a}^{-1} \stackrel{\text { def }}{=}\left(\left.\operatorname{ad} a\right|_{\mathfrak{m}}\right)^{-1}$. As an immediate consequence of Proposition 3 we obtain

Proposition 6. The pair $\left(A^{G}, \eta^{t}(\mathfrak{g}, a)\right)$ is Kronecker at a point $x \in R(\mathfrak{m})$ if and only if

(1) $\operatorname{dim}_{\mathbb{C}}\left(\left(\mathfrak{g}^{\mathbb{C}}\right)^{x+\lambda a}\right)_{\mathfrak{m}^{\mathbb{C}}}=r(\mathfrak{m})$ for each $\lambda \in \mathbb{C}$;

(2) $\operatorname{dim}_{\mathbb{C}} \operatorname{ker} B_{x}^{\mathrm{si}}=r(\mathfrak{m})$. 
Let $O^{\mathrm{Kr}}(\mathfrak{m})$ be a set of all points $x \in R(\mathfrak{m})$ for which conditions (1) and (2) of Proposition 6 hold. By Proposition 3 this set is a Zariski open (possibly empty) subset of $\mathfrak{m}$.

Denote by $I(\mathfrak{g})$ the space of all $\operatorname{Ad}(G)$-invariant polynomials on $\mathfrak{g}$. If $h \in I(\mathfrak{g})$ then it is clear that the function $h^{\lambda}: \mathfrak{g} \rightarrow \mathbb{R}, h^{\lambda}(y)=h(y+\lambda a), \lambda \in \mathbb{R}$, is $\operatorname{Ad}(K)$ invariant on $\mathfrak{g}$. Therefore the set $\mathcal{F}(\mathfrak{g}, \mathfrak{m})=\left\{\left.h^{\lambda}\right|_{\mathfrak{m}}, h \in I(\mathfrak{g}), \lambda \in \mathbb{R}\right\}$ is a subset of $A_{\mathfrak{m}}^{K}=A^{G}$ (of $G$-invariant functions on $T(G / K)$ ). The following assertion was proved in [MP] (see Proposition 3.6, Lemma 3.3 and Theorem 3.9).

Theorem $7([\mathrm{MP}])$. The Zariski open subset $O^{\mathrm{Kr}}(\mathfrak{m}) \subset R(\mathfrak{m})$ of $\mathfrak{m}$ is nonempty. The set of functions $\mathcal{F}(\mathfrak{g}, \mathfrak{m})$ is a complete involutive subset of the Poisson algebra $\left(A^{G}, \eta^{0}\right)$. Moreover, for each point $x \in O^{\mathrm{Kr}}(\mathfrak{m}) \subset R(\mathfrak{m})$ the space $\left\{\operatorname{grad}_{\mathfrak{m}} f(x), f \in\right.$ $\mathcal{F}(\mathfrak{g}, \mathfrak{m})\} \subset \mathfrak{m}(x)$ is a maximal isotropic subspace of $\mathfrak{m}(x)$.

By Theorem 7 the set

$$
\begin{aligned}
Q_{a}(\mathfrak{m}) & =\left\{x \in R(\mathfrak{m}): \operatorname{dim}_{\mathbb{C}} \operatorname{ker} B_{x}^{\mathrm{si}}=r(\mathfrak{m})\right\} \\
& =\left\{x \in R(\mathfrak{m}): \operatorname{dim}\left(\mathfrak{m}(x) \cap\left(\operatorname{ad}_{a}^{-1} \operatorname{ad} x(\mathfrak{k})\right)\right)=r(\mathfrak{m})\right\}
\end{aligned}
$$

contains the dense open subset $O^{\mathrm{Kr}}(\mathfrak{m}) \subset \mathfrak{m}$, in particular,

$$
r(\mathfrak{m})=\min _{x \in R(\mathfrak{m})} \operatorname{dim}\left(\mathfrak{m}(x) \cap\left(\operatorname{ad}_{a}^{-1} \operatorname{ad} x(\mathfrak{k})\right)\right) .
$$

Since $\mathfrak{m}(x)=(\operatorname{ad} x(\mathfrak{k}))^{\perp}$ in $\mathfrak{m}$ and $\operatorname{dim} \mathfrak{m}(x)$ is constant for $x \in R(\mathfrak{m})$, the set $Q_{a}(\mathfrak{m}) \subset R(\mathfrak{m})$ is a nonempty Zariski open subset of $\mathfrak{m}$. Put

$$
M_{a}(\mathfrak{m})=\left\{x \in \mathfrak{m}: \operatorname{dim}_{\mathbb{C}}\left(\mathfrak{g}^{\mathbb{C}}\right)^{x+\lambda a}=q(\mathfrak{m}) \text { for each } \lambda \in \mathbb{C}\right\} .
$$

Then

$$
O^{\mathrm{Kr}}(\mathfrak{m})=Q_{a}(\mathfrak{m}) \cap M_{a}(\mathfrak{m})
$$

because $Q_{a}(\mathfrak{m}) \subset R(\mathfrak{m})$ and $\left(\mathfrak{k}^{\mathbb{C}}\right)^{x+\lambda a}=\left(\mathfrak{k}^{\mathbb{C}}\right)^{x}$ for each $\lambda \in \mathbb{C}$.

Lemma 8. The set $M_{a}(\mathfrak{m})$ is a Zariski open subset of $\mathfrak{m}$.

Proof. The assertion of the lemma in more general form was used in the paper [BJ3, $\S 3.4]$ (but without any proof). For completeness we will prove this lemma here.

Let $V=\mathfrak{m}^{\mathbb{C}} \oplus \mathbb{C} a \subset \mathfrak{g}^{\mathbb{C}}$. Since by $(9), \min _{x \in \mathfrak{m}^{\mathbb{C}}, \lambda \in \mathbb{C}} \operatorname{dim}_{\mathbb{C}}\left(\mathfrak{g}^{\mathbb{C}}\right)^{x+\lambda a}=q(\mathfrak{m})$, the set

$$
S=\left\{y \in V: \operatorname{dim}_{\mathbb{C}}\left(\mathfrak{g}^{\mathbb{C}}\right)^{y}>q(\mathfrak{m})\right\}
$$

is a Zariski closed subset of $V$. If $y \in S$, then any non-zero (complex) scalar multiple of $y$ is also an element of $S$, so that one can consider a closed subvariety $\mathbb{P} S$ in the projectivization $\mathbb{P} V$ of $V$.

Suppose that $M_{a}(\mathfrak{m})$ is nonempty and $x_{0} \in M_{a}(\mathfrak{m})$. Then by definition $\left(x_{0}+\right.$ $\mathbb{C} a) \cap S=\varnothing$. In other words, the intersection of the projective line $\mathbb{P}\left\langle x_{0}, a\right\rangle$ with the set $\mathbb{P} S$ is empty. Clearly, any nearby line $\mathbb{P}\langle x, a\rangle, x \in \mathfrak{m}^{\mathbb{C}}$ will also not intersect $\mathbb{P} S$. The set $M_{a}\left(\mathfrak{m}^{\mathbb{C}}\right)$ of all such $x \in \mathfrak{m}^{\mathbb{C}}$ is Zariski open in $\mathfrak{m}^{\mathbb{C}}$. Taking into account that $M_{a}(\mathfrak{m})=M_{a}\left(\mathfrak{m}^{\mathbb{C}}\right) \cap \mathfrak{m}$ we complete the proof. 


\subsection{Adjoint orbits and involutive automorphisms}

Let $\sigma$ be an involutive automorphism of $\mathfrak{g}$ and let $\mathfrak{g}=\widetilde{\mathfrak{g}} \oplus \mathfrak{g}^{\prime}$ be the decomposition of $\mathfrak{g}$ into the eigenspaces of $\sigma$ for the eigenvalues +1 and -1 respectively:

$$
[\tilde{\mathfrak{g}}, \mathfrak{\mathfrak { g }}] \subset \widetilde{\mathfrak{g}}, \quad\left[\mathfrak{g}^{\prime}, \mathfrak{g}^{\prime}\right] \subset \widetilde{\mathfrak{g}}, \quad\left[\tilde{\mathfrak{g}}, \mathfrak{g}^{\prime}\right] \subset \mathfrak{g}^{\prime}
$$

Denote by $\widetilde{G}$ the closed connected subgroup of $G$ with the Lie algebra $\widetilde{\mathfrak{g}}$. Fix some element $a \in \mathfrak{g}^{\prime}(\sigma(a)=-a)$ and consider the orbit $\widetilde{\mathcal{O}}=\operatorname{Ad}(\widetilde{G})(a)=\widetilde{G} / \widetilde{K}$ in $\mathfrak{g}$ through this element $a$. It is clear that $\widetilde{\mathcal{O}}$ is a submanifold ( $\widetilde{G}$-suborbit) of the $G$-orbit $\mathcal{O}=G / K$ of $a$ and $\widetilde{K}=\widetilde{G} \cap K$. The form $\langle\cdot, \cdot\rangle$ determines a $\widetilde{G}$-invariant metric on $\widetilde{\mathcal{O}}$. This metric identifies the cotangent bundle $T^{*} \widetilde{\mathcal{O}}$ and the tangent bundle $T \widetilde{\mathcal{O}}$. Thus we can also talk on the canonical 2 -form $\widetilde{\Omega}$ on the manifold $T \widetilde{\mathcal{O}}$.

Since $\sigma(a)=-a$, the algebra $\mathfrak{k}=\mathfrak{g}^{a}$ is $\sigma$-invariant. Suppose that the form $\langle\cdot, \cdot\rangle=-\Phi_{\chi}$ is also $\sigma$-invariant (if $\mathfrak{g}$ is semi-simple its Killing form is invariant with respect to an arbitrary automorphism of $\mathfrak{g})$. Then $\sigma(\mathfrak{m})=\mathfrak{m}$ and we have in addition to (1) the following orthogonal decompositions of algebras $\mathfrak{g}, \widetilde{\mathfrak{g}}, \mathfrak{k}$ with respect to the form $\langle\cdot, \cdot\rangle$ :

$$
\left.\left.\mathfrak{g}=\widetilde{\mathfrak{k}} \oplus \mathfrak{k}^{\prime} \oplus \widetilde{\mathfrak{m}} \oplus \mathfrak{m}^{\prime}, \quad \widetilde{\mathfrak{g}}=\widetilde{\mathfrak{k}} \oplus \widetilde{\mathfrak{m}}, \quad \widetilde{[\mathfrak{k}}, \widetilde{\mathfrak{m}}\right] \subset \widetilde{\mathfrak{m}}, \quad \widetilde{\mathfrak{k}}, \mathfrak{m}^{\prime}\right] \subset \mathfrak{m}^{\prime}, \quad \mathfrak{k}=\widetilde{\mathfrak{k}} \oplus \mathfrak{k}^{\prime},
$$

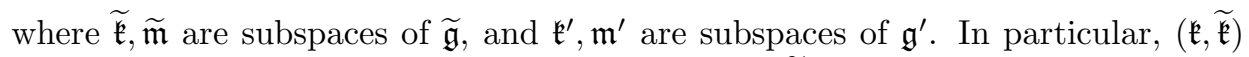
is a symmetric pair of compact Lie algebras, i.e., $\widetilde{\mathfrak{k}}$ is the fixed point set of the involutive automorphism $\left.\sigma\right|_{\mathfrak{k}}$.

Since ker ad $a=\mathfrak{k}$ and $\mathfrak{m}=\mathfrak{k}^{\perp}$ in $\mathfrak{g}$, then $\operatorname{ad} a(\mathfrak{m})=\mathfrak{m}$ and the operator $\left.\operatorname{ad} a\right|_{\mathfrak{m}}$ : $\mathfrak{m} \rightarrow \mathfrak{m}$ is invertible. Moreover, for $\mathfrak{m}^{\prime} \subset \mathfrak{m}$ and $\widetilde{\mathfrak{m}} \subset \mathfrak{m}$ we have

$\operatorname{ad} a\left(\mathfrak{m}^{\prime}\right) \subset\left[\mathfrak{g}^{\prime}, \mathfrak{m}^{\prime}\right] \cap \mathfrak{m} \subset \widetilde{\mathfrak{g}} \cap \mathfrak{m} \subset \widetilde{\mathfrak{m}}, \quad$ and $\quad \operatorname{ad} a(\widetilde{\mathfrak{m}}) \subset\left[\mathfrak{g}^{\prime}, \widetilde{\mathfrak{m}}\right] \cap \mathfrak{m} \subset \mathfrak{g}^{\prime} \cap \mathfrak{m} \subset \mathfrak{m}^{\prime}$ and therefore $\operatorname{dim} \tilde{\mathfrak{m}}=\operatorname{dim} \mathfrak{m}^{\prime}$. Since $\operatorname{ker}\left(\left.\operatorname{ad} a\right|_{\mathfrak{m}}\right)=0$, we have

$$
\operatorname{ad} a\left(\mathfrak{m}^{\prime}\right)=\widetilde{\mathfrak{m}}, \quad \text { and } \quad \operatorname{ad} a(\widetilde{\mathfrak{m}})=\mathfrak{m}^{\prime}
$$

Similarly as in the case of the pair $(\mathfrak{g}, \mathfrak{k})$, for any $x \in \widetilde{\mathfrak{m}}$ define the subspace $\widetilde{\mathfrak{m}}(x) \subset \mathfrak{m}$ putting

$$
\widetilde{\mathfrak{m}}(x) \stackrel{\text { def }}{=}\{y \in \widetilde{\mathfrak{m}}:[x, y] \in \widetilde{\mathfrak{m}}\}=\{y \in \widetilde{\mathfrak{m}}:\langle y, \operatorname{ad} x(\widetilde{\mathfrak{k}})\rangle=0\}
$$

Then, in particular, ad $x(\widetilde{\mathfrak{m}}(x)) \subset \widetilde{\mathfrak{m}}$ and $\widetilde{\mathfrak{m}}(x) \oplus \operatorname{ad} x(\widetilde{\mathfrak{k}})=\widetilde{\mathfrak{m}}$.

Let $A^{\widetilde{G}}$ (resp. $A_{\widetilde{\mathfrak{m}}}^{\widetilde{K}}$ ) be the set of all $\widetilde{G}$-invariant (resp. $\operatorname{Ad}(\widetilde{K})$-invariant) functions on $T(\widetilde{G} / \widetilde{K})$ (resp. on $\widetilde{\mathfrak{m}}$ ). The Poisson bracket of two functions $\widetilde{f}_{1}, \widetilde{f}_{2} \in A_{\widetilde{\mathfrak{m}}}^{\widetilde{K}}$ with respect to the canonical Poisson structure $\widetilde{\eta}^{\text {can }}$ (determined by the canonical 2 -form $\widetilde{\Omega}$ on $T \widetilde{\mathcal{O}}$ ) has the form (see $(2))$ :

$$
\left\{\widetilde{f}_{1}, \widetilde{f}_{2}\right\}^{\operatorname{can}}(x)=-\left\langle x,\left[\operatorname{grad}_{\widetilde{\mathfrak{m}}} \widetilde{f}_{1}(x), \operatorname{grad}_{\widetilde{\mathfrak{m}}} \widetilde{f}_{2}(x)\right]\right\rangle, \quad x \in \widetilde{\mathfrak{m}} .
$$


Note that by $\operatorname{Ad}(\widetilde{K})$-invariance of the function $\widetilde{f}_{k}$, the vector $\operatorname{grad}_{\widetilde{\mathfrak{m}}} \widetilde{f}_{k}(x), k=1,2$, belongs to the subspace $\widetilde{\mathfrak{m}}(x) \subset \widetilde{\mathfrak{m}}$.

Since $\sigma(a)=-a$, the center $\mathfrak{z}=\mathfrak{z}(\mathfrak{k})$ of the reductive Lie algebra $\mathfrak{k}=\mathfrak{g}^{a}$ is $\sigma$-invariant, i.e., $\mathfrak{z}=\widetilde{\mathfrak{z}} \oplus \mathfrak{z}^{\prime}$, where $\widetilde{\mathfrak{z}}=\mathfrak{z} \cap \tilde{\mathfrak{g}}, \mathfrak{z}^{\prime}=\mathfrak{z} \cap \mathfrak{g}^{\prime}$. It is clear that $a \in \mathfrak{z}^{\prime}$. Then for each element $b \in \mathfrak{z}^{\prime}$ we can consider the endomorphism $\varphi_{a, b}: \mathfrak{g} \rightarrow \mathfrak{g}$ on $\mathfrak{g}$ putting $\varphi_{a, b}(x)=\operatorname{ad}_{a}^{-1}([b, x])$ for $x \in \mathfrak{m}$ and $\varphi_{a, b}(z)=z$ for $z \in \mathfrak{k}$, where, recall, $\operatorname{ad}_{a}^{-1} \stackrel{\text { def }}{=}\left(\left.\operatorname{ad} a\right|_{\mathfrak{m}}\right)^{-1}$. Remark that $\varphi_{a, b}(\mathfrak{m}) \subset \mathfrak{m}$ because $[\mathfrak{k}, \mathfrak{m}] \subset \mathfrak{m}$. Moreover, $\varphi_{a, b}(\widetilde{\mathfrak{m}}) \subset \widetilde{\mathfrak{m}}$ and $\varphi_{a, b}\left(\mathfrak{m}^{\prime}\right) \subset \mathfrak{m}^{\prime}$ because $a, b \in \mathfrak{g}^{\prime}$ (see also (16)).

It is clear that the endomorphism $\varphi_{a, b}$ is symmetric and the $\operatorname{group} \operatorname{Ad}(K)$ commutes elementwise with $\varphi_{a, b}$ on $\mathfrak{m}$. Therefore, the operator $\left.\varphi_{a, b}\right|_{\mathfrak{m}}$ is also symmetric and the group $\operatorname{Ad}(\widetilde{K})$ commutes elementwise with $\varphi_{a, b}$ on $\widetilde{\mathfrak{m}}(K$ is connected). Therefore the function $\widetilde{H}_{a, b}(x)=\frac{1}{2}\left\langle x, \varphi_{a, b}(x)\right\rangle, x \in \widetilde{\mathfrak{m}}$, is $\operatorname{Ad}(\widetilde{K})$ invariant. Then $\widetilde{H}_{a, b}$ (as a function on $T(\widetilde{G} / \widetilde{K})$ from the set $A^{\widetilde{G}}=A_{\widetilde{\mathfrak{m}}}^{\widetilde{K}}$ ) is a Hamiltonian function of the geodesic flow of some pseudo-Riemannian metric on $\widetilde{G} / \widetilde{K}$ if $\left.\varphi_{a, b}\right|_{\tilde{m}}$ is non-degenerate.

Consider the space $I(\mathfrak{g})$ of all $\operatorname{Ad}(G)$-invariant polynomials on $\mathfrak{g}$. As we remarked in the previous subsection, for each $h \in I(\mathfrak{g})$ the function $h^{\lambda}: \mathfrak{g} \rightarrow \mathbb{R}$, $h^{\lambda}(y)=h(y+\lambda a), \lambda \in \mathbb{R}$, is an $\operatorname{Ad}(K)$-invariant function on $\mathfrak{g}$. Therefore the set $\mathcal{F}(\mathfrak{g}, \mathfrak{m})=\left\{\left.h^{\lambda}\right|_{\mathfrak{m}}, h \in I(\mathfrak{g}), \lambda \in \mathbb{R}\right\}$ is a subset of $A_{\mathfrak{m}}^{K}=A^{G}$ (of $G$-invariant function on $T(G / K))$ and the set $\mathcal{F}(\mathfrak{g}, \widetilde{\mathfrak{m}})=\left\{\left.h^{\lambda}\right|_{\widetilde{\mathfrak{m}}}, h \in I(\mathfrak{g}), \lambda \in \mathbb{R}\right\}$ is a subset of $A_{\widetilde{\mathfrak{m}}}^{\widetilde{K}}=A^{\widetilde{G}}$ (of $\widetilde{G}$-invariant function on $\left.T(\widetilde{G} / \widetilde{K})\right)$. Put $H^{\lambda}=\left.h^{\lambda}\right|_{\mathfrak{m}}$ and $\widetilde{H}^{\lambda}=\left.h^{\lambda}\right|_{\widetilde{\mathfrak{m}}}$. The following lemma follows easily from the results of [MF1] (see also [TF, Chap. 6, 16, Lem.] or [DGJ1, Sec. 3]).

Lemma 9 ([DGJ1]). For any functions $h_{1}, h_{2}, h \in I(\mathfrak{g})$ and arbitrary parameters $\lambda_{1}, \lambda_{2}, \lambda \in \mathbb{R}$ we have $\left\{\widetilde{H}_{1}^{\lambda_{1}}, \widetilde{H}_{2}^{\lambda_{2}}\right\}^{\text {can }}=0$ and $\left\{\widetilde{H}^{\lambda}, \widetilde{H}_{a, b}\right\}^{\text {can }}=0$.

Proof. Mainly to fix notations we will prove this lemma here. Since $\sigma$ is an automorphism of $\mathfrak{g}$ and $\operatorname{Ad}(G)$ is a normal subgroup of $\operatorname{Aut}(\mathfrak{g})$, we have $f=h \circ \sigma \in I(\mathfrak{g})$ if $h \in I(\mathfrak{g})$. However,

$$
2 \operatorname{grad}_{\mathfrak{g}} h(x+\lambda a)=\operatorname{grad}_{\mathfrak{g}} h(x+\lambda a)+\operatorname{grad}_{\mathfrak{g}} f(x-\lambda a) \quad \text { for any } \quad x \in \widetilde{\mathfrak{g}},
$$

because $\sigma(a)=-a$, and $\sigma(x)=x$. The five functions $h_{1}^{\lambda_{1}}, h_{2}^{\lambda_{2}}, f_{1}^{-\lambda_{1}}, f_{2}^{-\lambda_{2}}$ and $h_{a, b}$ commute pairwise on $\mathfrak{g} \simeq \mathfrak{g}^{*}$ with respect to the standard (linear) Lie-Poisson bracket on $\mathfrak{g}[\mathrm{MF} 1]$. This means that for any pair of functions $F_{1}, F_{2}$ from this set we have

Then by (18)

$$
\left\langle x,\left[\operatorname{grad}_{\mathfrak{g}} F_{1}(x), \operatorname{grad}_{\mathfrak{g}} F_{2}(x)\right]\right\rangle=0 \quad \text { for all } \quad x \in \mathfrak{g} .
$$

$$
\left.\left\langle x, \operatorname{grad}_{\mathfrak{\mathfrak { g }}} h_{1}^{\lambda_{1}}(x), \operatorname{grad}_{\tilde{\mathfrak{g}}} h_{2}^{\lambda_{2}}(x)\right]\right\rangle=0 \quad \text { for all } \quad x \in \widetilde{\mathfrak{m}} \subset \mathfrak{g} .
$$

Now taking into account that $\left(\operatorname{grad}_{\mathfrak{\mathfrak { g }}} h_{j}^{\lambda_{j}}(x)\right)_{\widetilde{\mathfrak{m}}}=\operatorname{grad}_{\widetilde{\mathfrak{m}}} \widetilde{H}_{j}^{\lambda_{j}}(x) \in \widetilde{\mathfrak{m}}(x),[x, \widetilde{\mathfrak{m}}(x)] \subset$ $\widetilde{\mathfrak{m}}$ for $x \in \widetilde{\mathfrak{m}}$ and $\widetilde{\mathfrak{m}} \perp \widetilde{\mathfrak{k}}$, we obtain that

$$
\left\langle x,\left[\left(\operatorname{grad}_{\mathfrak{\mathfrak { g }}} h_{1}^{\lambda_{1}}(x)\right)_{\widetilde{\mathfrak{m}}},\left(\operatorname{grad}_{\widetilde{\mathfrak{g}}} h_{2}^{\lambda_{2}}(x)\right)_{\widetilde{\mathfrak{m}}}\right]\right\rangle=0,
$$

i.e., $\left\{\widetilde{H}_{1}^{\lambda_{1}}, \widetilde{H}_{2}^{\lambda_{2}}\right\}^{\text {can }}(x)=0$. Similarly we can show that $\left\{\widetilde{H}^{\lambda}, \widetilde{H}_{a, b}\right\}^{\text {can }}=0$. 
As follows from the lemma above, the set $\mathcal{F}(\mathfrak{g}, \widetilde{\mathfrak{m}})$ is an involutive subset of $\left(A_{\widetilde{\mathfrak{m}}}^{\widetilde{K}}, \widetilde{\eta}^{\text {can }}\right)$. Let us define the numbers $q(\widetilde{\mathfrak{m}}), p(\widetilde{\mathfrak{m}}), r(\widetilde{\mathfrak{m}})$ and the subset $R(\widetilde{\mathfrak{m}}) \subset \widetilde{\mathfrak{m}}$ similarly to the numbers $q(\mathfrak{m}), p(\mathfrak{m}), r(\mathfrak{m})$ and the subset $R(\mathfrak{m}) \subset \mathfrak{m}$, but for the pair of algebras $(\widetilde{\mathfrak{g}}, \widetilde{\mathfrak{k}})($ see $(4))$.

Let $\widetilde{\mu}^{\text {can }}: T(\widetilde{G} / \widetilde{K}) \rightarrow \widetilde{\mathfrak{g}}^{*}$ be the standard moment map associated with the natural action of $\widetilde{G}$ on $(T(\widetilde{G} / \widetilde{K}), \widetilde{\Omega}))$. The subset $\left\{\widetilde{h} \circ \widetilde{\mu}^{\text {can }}, \widetilde{h} \in \mathcal{E}\left(\widetilde{\mathfrak{g}}^{*}\right)\right\} \subset$ $\mathcal{E}(T(\widetilde{G} / \widetilde{K}))$ is a Poisson subalgebra of $\left(\mathcal{E}(T(\widetilde{G} / \widetilde{K})), \widetilde{\eta}^{\text {can }}\right)$. Since the Lie group $\widetilde{G}$ is compact, this subalgebra contains some complete involutive subset of functions $\mathcal{H}\left(\widetilde{\mathfrak{g}}^{*}\right)[\mathrm{Br}],[\mathrm{My} 1]$. By definition of the moment map $\widetilde{\mu}^{\text {can }}$, the union of the sets $\mathcal{H}\left(\widetilde{\mathfrak{g}}^{*}\right)$ and $\mathcal{F}(\mathfrak{g}, \widetilde{\mathfrak{m}}) \subset A_{\widetilde{\mathfrak{m}}}^{\widetilde{K}}=A^{\widetilde{G}}$ is an involutive subset of the Poisson algebra $\left(\mathcal{E}(T(\widetilde{G} / \widetilde{K})), \widetilde{\eta}^{\text {can }}\right)$.

Theorem 10. Suppose that the Zariski open subset $O^{\mathrm{Kr}}(\mathfrak{m}) \cap \widetilde{\mathfrak{m}}$ of $\widetilde{\mathfrak{m}}$ is nonempty. Then the set $\mathcal{F}(\mathfrak{g}, \widetilde{\mathfrak{m}})$ is a complete involutive subset of the algebra $\left(A_{\widetilde{\mathfrak{m}}}^{\widetilde{K}}, \widetilde{\eta}^{\text {can }}\right)$. Moreover, the set $\mathcal{H}\left(\widetilde{\mathfrak{g}}^{*}\right) \cup \mathcal{F}(\mathfrak{g}, \widetilde{\mathfrak{m}})$ is a complete involutive subset of the algebra $\left(\mathcal{E}(T(\widetilde{G} / \widetilde{K})), \widetilde{\eta}^{\text {can }}\right)$ and the functions from this set are integrals for the Hamiltonian flow with the Hamiltonian function $\widetilde{H}_{a, b}$ on $T(\widetilde{G} / \widetilde{K})$.

Proof. Fix some point $x \in O^{\mathrm{Kr}}(\mathfrak{m}) \cap R(\widetilde{\mathfrak{m}}) \subset \widetilde{\mathfrak{m}}$. Put

$$
\begin{aligned}
L_{x} & =\left\{\operatorname{grad}_{\mathfrak{m}} f(x), f \in \mathcal{F}(\mathfrak{g}, \mathfrak{m})\right\} \subset \mathfrak{m}(x) \subset \mathfrak{m}, \\
\widetilde{L}_{x} & =\left\{\operatorname{grad}_{\widetilde{\mathfrak{m}}} \widetilde{f}(x), \widetilde{f} \in \mathcal{F}(\mathfrak{g}, \widetilde{\mathfrak{m}})\right\} \subset \widetilde{\mathfrak{m}}(x) \subset \widetilde{\mathfrak{m}}
\end{aligned}
$$

It is evident that $\widetilde{L}_{x}=\left(L_{x}\right)_{\widetilde{\mathfrak{m}}}$, where $(\cdot)_{\widetilde{\mathfrak{m}}}$ denotes the projection onto $\widetilde{\mathfrak{m}}$ along $\mathfrak{m}^{\prime}$ in $\mathfrak{m}=\widetilde{\mathfrak{m}} \oplus \mathfrak{m}^{\prime}$. Moreover, since $x \in \widetilde{\mathfrak{m}}$ and $\mathfrak{k}=\widetilde{\mathfrak{k}} \oplus \mathfrak{k}^{\prime}$, we have ad $x(\mathfrak{k})=$ $\operatorname{ad} x(\widetilde{\mathfrak{k}}) \oplus \operatorname{ad} x\left(\mathfrak{k}^{\prime}\right)$, where $\operatorname{ad} x(\widetilde{\mathfrak{k}}) \subset \widetilde{\mathfrak{m}}$ and $\operatorname{ad} x\left(\mathfrak{k}^{\prime}\right) \subset \mathfrak{m}^{\prime}$, and therefore

$$
\mathfrak{m}(x)=\widetilde{\mathfrak{m}}(x) \oplus\left(\mathfrak{m}(x) \cap \mathfrak{m}^{\prime}\right) .
$$

By Lemma 9 the space $\widetilde{L}_{x}$ is an isotropic subspace of $\widetilde{\mathfrak{m}}(x)$ with respect to the form $\widetilde{B}_{x}:\left(y_{1}, y_{2}\right) \mapsto-\left\langle x,\left[y_{1}, y_{2}\right]\right\rangle$ on $\widetilde{\mathfrak{m}}(x)$ associated with Poisson bracket (17). Therefore, in order to prove the theorem it is sufficient to show that this subspace is maximal isotropic.

To this end, suppose that $\left\langle x,\left[y, \widetilde{L}_{x}\right]\right\rangle=0$ for some $y \in \widetilde{\mathfrak{m}}(x)$. Then $\left\langle[x, y], \widetilde{L}_{x}\right\rangle=$ 0 by invariance of the form $\langle\cdot, \cdot\rangle$. Taking into account that $[x, \widetilde{\mathfrak{m}}(x)] \subset \widetilde{\mathfrak{m}}$ by definition and $\widetilde{\mathfrak{m}} \perp \mathfrak{m}^{\prime}$, we obtain that

$$
0=\left\langle[x, y], \widetilde{L}_{x}\right\rangle=\left\langle[x, y], L_{x}\right\rangle=\left\langle x,\left[y, L_{x}\right]\right\rangle .
$$

But $y \in \mathfrak{m}(x)$ by (19). Also, by Theorem 7 the space $L_{x}$ is a maximal isotropic in $\mathfrak{m}(x)$ and, consequently, $y \in L_{x}$. Then $y \in \widetilde{L}_{x}$, because $y \in \widetilde{\mathfrak{m}}$ and $L_{x} \cap \widetilde{\mathfrak{m}} \subset$ $\left(L_{x}\right)_{\mathfrak{m}}=\widetilde{L}_{x}$. In other words, $\widetilde{L}_{x}$ is a maximal isotropic subspace in $\widetilde{\mathfrak{m}}(x)$ with respect to the form $\widetilde{B}_{x}$ defined by $(17)$ on $\widetilde{\mathfrak{m}}(x)$.

Therefore the set $\mathcal{F}(\mathfrak{g}, \widetilde{\mathfrak{m}})$ is a complete involutive subset of the algebra $A_{\widetilde{\mathfrak{m}}}^{\widetilde{K}}=$ $A^{\widetilde{G}}$ with respect to the canonical Poisson structure on $(T(\widetilde{G} / \widetilde{K}), \widetilde{\Omega})$. Then by $[\mathrm{BJ} 1$, 
Lem. 3] (see also $[\mathrm{My} 3, \S 2],[\mathrm{Pa}])$ the set $\mathcal{H}\left(\widetilde{\mathfrak{g}}^{*}\right) \cup \mathcal{F}(\mathfrak{g}, \widetilde{\mathfrak{m}})$ is a complete involutive subset of the Poisson algebra $\left(\mathcal{E}(T(\widetilde{G} / \widetilde{K})), \widetilde{\eta}^{\text {can }}\right)$ on the manifold $(T(\widetilde{G} / \widetilde{K}), \widetilde{\Omega})$. By Lemma 9 the functions from the set $\mathcal{F}(\mathfrak{g}, \widetilde{\mathfrak{m}})$ are integrals of the Hamiltonian flow with the Hamiltonian function $\widetilde{H}_{a, b}$ on $T(\widetilde{G} / \widetilde{K})$. The functions from the set $\mathcal{H}\left(\widetilde{\mathfrak{g}}^{*}\right)$ are also integrals for this flow because $\widetilde{H}_{a, b} \subset A^{\widetilde{G}}$.

Remark 11. First of all note that the Hamiltonian flow of the function $\widetilde{H}_{a, a}$ (with $b=a)$ coincides with the geodesic flow determined by the Riemannian metric $\langle\cdot, \cdot\rangle$ on $\widetilde{G} / \widetilde{K}$. The Hamiltonian function $\widetilde{H}_{a, b}$ on $T \widetilde{\mathcal{O}}$ is the restriction of the Hamiltonian function $H_{a, b}(x)=\left\langle x, \varphi_{a, b}(x)\right\rangle / 2, x \in \mathfrak{m}$ (considered as a $G$-invariant function on $T \mathcal{O}$ from the set $A^{G}=A_{\mathfrak{m}}^{K}$ ). Moreover, the function $H_{a, b}$ is associated with the (pseudo)-Riemannian metric $g_{a, b}$ on $\mathcal{O}$ (defined by the symmetric bilinear form $\left.(x, y) \mapsto\left\langle x, \varphi_{a, b}^{-1}(y)\right\rangle, x, y \in \mathfrak{m}\right)$ and $\widetilde{H}_{a, b}$ with its restriction $\widetilde{g}_{a, b}=\left.g_{a, b}\right|_{\widetilde{\mathcal{O}}}$. Since $\operatorname{grad}_{\mathfrak{m}} H_{a, b}(x)=\operatorname{grad}_{\widetilde{\mathfrak{m}}} \widetilde{H}_{a, b}(x)=\varphi_{a, b}(x) \in \widetilde{\mathfrak{m}}$ for each $x \in \widetilde{\mathfrak{m}}$, it is easy to verify that the Hamiltonian vector field $X_{H_{a, b}}$ of $H_{a, b}$ on $T \mathcal{O}$ is tangent to the submanifold $T \widetilde{\mathcal{O}} \subset T \mathcal{O}$ (using, for instance, the exact expression for $X_{H_{a, b}}$ on $T \mathcal{O}[\mathrm{MP},(3.6)])$. As follows from Proposition $4,(T \widetilde{\mathcal{O}}, \widetilde{\Omega})$ is a symplectic submanifold of $(T \mathcal{O}, \Omega)$. Therefore the Hamiltonian vector field $X_{\widetilde{H}_{a, b}}$ of $\widetilde{H}_{a, b}$ on $T \widetilde{\mathcal{O}}$ coincides with the restriction $\left.X_{H_{a, b}}\right|_{T \widetilde{\mathcal{O}}}$ and, consequently, the suborbit $\left(\widetilde{\mathcal{O}}, \widetilde{g}_{a, b}\right)$ is a totally geodesic submanifold of the orbit $\left(\mathcal{O}, g_{a, b}\right)$. Taking into account that the geodesic flow of the metric $g_{a, b}$ on $T \mathcal{O}$ is integrable (see [MP, Thm. 3.10] or [BJ3]), Theorem 10 above is an illustration to the phenomenon of a complete integrability of the geodesic flow on a totally geodesic submanifold of a manifold with the integrable geodesic flow.

Remark 12. Let $\mathfrak{g}_{1}$ be some $\sigma$-invariant subalgebra of $\mathfrak{g}$ containing its maximal semisimple ideal $\mathfrak{g}_{s}$. Assume that the connected subgroup $G_{1} \subset G$ with the Lie algebra $\mathfrak{g}_{1}$ is closed. Denote by $a_{1} \in \mathfrak{g}_{1}$ the orthogonal projection of the element $a \in \mathfrak{g}$. Put $\mathfrak{k}_{1}=\mathfrak{g}_{1}^{a_{1}}\left(\mathfrak{k}=\mathfrak{g}^{a}\right)$. Since the algebra $\mathfrak{k}=\mathfrak{g}^{a}$ contains the center $\mathfrak{z}(\mathfrak{g})$ of $\mathfrak{g}$, the space $\mathfrak{m}=\mathfrak{k}^{\perp}$ is a subspace of the ideal $\mathfrak{g}_{s}$ and the space $\mathfrak{m}_{1}=\mathfrak{k}_{1}^{\perp} \subset \mathfrak{g}_{1}$ coincides with $\mathfrak{m}$. Consequently, the bi-Poisson structures $\eta^{t}(\mathfrak{g}, a)$ and $\eta^{t}\left(\mathfrak{g}_{1}, a_{1}\right)$ are either Kronecker or non-Kronecker simultaneously at a point $x \in R(\mathfrak{m}) \cap \widetilde{\mathfrak{m}}$ if $R(\mathfrak{m}) \cap \widetilde{\mathfrak{m}} \neq \varnothing$.

\subsection{Conditional reduction theorem}

We will use the notation introduced in the previous subsection. Suppose that the set $R(\mathfrak{m}) \cap \widetilde{\mathfrak{m}}$ is nonempty and that $x_{0}$ is a common element of this set and the set $R(\widetilde{\mathfrak{m}})$. Let $\mathfrak{g}_{0}$ be the centralizer of $\mathfrak{k}^{x_{0}}$ in $\mathfrak{g}$. Put $\mathfrak{k}_{0}=\mathfrak{k} \cap \mathfrak{g}_{0}$ and $\mathfrak{m}_{0}=\mathfrak{m} \cap \mathfrak{g}_{0}$. Since $\sigma\left(x_{0}\right)=x_{0}$ and $\sigma(\mathfrak{k})=\mathfrak{k}$, we have $\sigma\left(\mathfrak{k}^{x_{0}}\right)=\mathfrak{k}^{x_{0}}$. Therefore the spaces $\mathfrak{g}_{0}, \mathfrak{k}_{0}$ and $\mathfrak{m}_{0}$ are $\sigma$-invariant, i.e.,

$$
\mathfrak{g}_{0}=\mathfrak{k}_{0} \oplus \mathfrak{m}_{0}, \quad \widetilde{\mathfrak{g}}_{0}=\widetilde{\mathfrak{k}}_{0} \oplus \widetilde{\mathfrak{m}}_{0}, \quad \text { where } \widetilde{\mathfrak{g}}_{0}=\mathfrak{g}_{0} \cap \widetilde{\mathfrak{g}}, \widetilde{\mathfrak{k}}_{0}=\mathfrak{k}_{0} \cap \widetilde{\mathfrak{k}}, \widetilde{\mathfrak{m}}_{0}=\mathfrak{m}_{0} \cap \widetilde{\mathfrak{m}} .
$$

By Proposition 5, $x_{0} \in R\left(\mathfrak{m}_{0}\right), a \in \mathfrak{g}_{0}$ and $\mathfrak{k}_{0}=\mathfrak{g}_{0}^{a}\left(\mathfrak{k}=\mathfrak{g}^{a}\right)$. Now we will prove the following conditional reduction theorem which reduces the pair $\left(\mathfrak{g}, \mathfrak{k}=\mathfrak{g}^{a}\right)$ to the pair $\left(\mathfrak{g}_{0}, \mathfrak{k}_{0}=\mathfrak{g}_{0}^{a}\right)$ if the condition $R(\mathfrak{m}) \cap \widetilde{\mathfrak{m}} \neq \varnothing$ holds. To this end, define the sets $O^{\mathrm{Kr}}\left(\mathfrak{m}_{0}\right), Q_{a}\left(\mathfrak{m}_{0}\right)$ and $M_{a}\left(\mathfrak{m}_{0}\right)$ similarly to the sets $O^{\mathrm{Kr}}(\mathfrak{m}), Q_{a}(\mathfrak{m})$ and $M_{a}(\mathfrak{m})$, but for the pair of algebras $\left(\mathfrak{g}_{0}, \mathfrak{k}_{0}\right)$ (see (14), (11) and (13)). 
Theorem 13. Suppose that $R(\mathfrak{m}) \cap \widetilde{\mathfrak{m}} \neq \varnothing$ and choose any point $x_{0} \in R(\mathfrak{m}) \cap R(\widetilde{\mathfrak{m}})$. The set $O^{\mathrm{Kr}}(\mathfrak{m}) \cap \widetilde{\mathfrak{m}}$ is nonempty if the set $O^{\mathrm{Kr}}\left(\mathfrak{m}_{0}\right) \cap \widetilde{\mathfrak{m}}_{0}$ is also. In particular, if $O^{\mathrm{Kr}}\left(\mathfrak{m}_{0}\right) \cap \widetilde{\mathfrak{m}}_{0} \neq \varnothing$ then the set $\mathcal{F}(\mathfrak{g}, \widetilde{\mathfrak{m}})$ is a complete involutive subset of the $\operatorname{algebra}\left(A_{\widetilde{\mathfrak{m}}}^{\widetilde{K}}, \widetilde{\eta}^{\text {can }}\right)$.

Proof. Assume that $O^{\mathrm{Kr}}\left(\mathfrak{m}_{0}\right) \cap \widetilde{\mathfrak{m}}_{0} \neq \varnothing$. By definition, $x_{0} \in R(\mathfrak{m}) \cap R(\widetilde{\mathfrak{m}})$ and $x_{0} \in \widetilde{\mathfrak{m}}_{0}$ by the construction above. Therefore the set $R(\mathfrak{m}) \cap R(\widetilde{\mathfrak{m}}) \cap O^{\mathrm{Kr}}\left(\mathfrak{m}_{0}\right) \cap \widetilde{\mathfrak{m}}_{0}$ is a nonempty Zariski open subset of $\widetilde{\mathfrak{m}}_{0}$. Thus the set $R(\mathfrak{m}) \cap R(\widetilde{\mathfrak{m}}) \cap O^{\mathrm{Kr}}\left(\mathfrak{m}_{0}\right) \cap R\left(\widetilde{\mathfrak{m}}_{0}\right)$ is also nonempty. Fix some element $x$ belonging to this set. To prove the theorem, it is sufficient to show that $x \in O^{\mathrm{Kr}}(\mathfrak{m})$.

Since $x \in R(\mathfrak{m}) \cap \mathfrak{m}_{0}$, by Proposition 5 (1) we have that $\mathfrak{m}(x)=\mathfrak{m}_{0}(x)$. Then by relations (10) the two bi-Poisson structures $\eta(\mathfrak{g}, a)$ and $\eta\left(\mathfrak{g}_{0}, a\right)$ determine on the space $\mathfrak{m}(x)=\mathfrak{m}_{0}(x)$ the same families of skew-symmetric operators. In other words, at the point $x \in R(\mathfrak{m}) \cap R\left(\mathfrak{m}_{0}\right)$ these bi-Poisson structures are Kronecker simultaneously. Thus $x \in O^{\mathrm{Kr}}(\mathfrak{m}) \cap \widetilde{\mathfrak{m}}$.

As follows from Theorem 10, we have to establish when the set $O^{\mathrm{Kr}}(\mathfrak{m}) \cap \tilde{\mathfrak{m}}$ is nonempty. Since the subsets $Q_{a}(\mathfrak{m}) \subset \mathfrak{m}$ and $M_{a}(\mathfrak{m}) \subset \mathfrak{m}$ are Zariski open (see (12) and Lemma 8), we obtain by (14) that

$$
O^{\mathrm{Kr}}(\mathfrak{m}) \cap \widetilde{\mathfrak{m}} \neq \varnothing \quad \Longleftrightarrow \quad Q_{a}(\mathfrak{m}) \cap \widetilde{\mathfrak{m}} \neq \varnothing \quad \text { and } \quad M_{a}(\mathfrak{m}) \cap \widetilde{\mathfrak{m}} \neq \varnothing
$$

By Proposition 5 (4) for any element $x \in R(\mathfrak{m}) \cap \mathfrak{m}_{0}$ its centralizer $\mathfrak{k}_{0}^{x_{0}}$ in $\mathfrak{k}_{0}$ is the center $\mathfrak{z}\left(\mathfrak{g}_{0}\right)$ of the Lie algebra $\mathfrak{g}_{0}$. As follows from Theorem 13 and relations $(20)$, to prove a completeness of the set $\mathcal{F}(\mathfrak{g}, \widetilde{\mathfrak{m}})$ it is sufficient to show that $Q_{a}\left(\mathfrak{m}_{0}\right) \cap \widetilde{\mathfrak{m}}_{0} \neq \varnothing$ and $M_{a}\left(\mathfrak{m}_{0}\right) \cap \widetilde{\mathfrak{m}}_{0} \neq \varnothing$. Therefore we will investigate the two open subsets $Q_{a}(\mathfrak{m}) \cap \widetilde{\mathfrak{m}}$ and $M_{a}(\mathfrak{m}) \cap \widetilde{\mathfrak{m}}$ in more detail in the following two subsections only in the case when for each element $x \in R(\mathfrak{m})$ its centralizer $\mathfrak{k}^{x}=\mathfrak{g}^{a} \cap \mathfrak{g}^{x}$ is the center $\mathfrak{z}(\mathfrak{g})$ of $\mathfrak{g}$.

\subsection{Necessary and sufficient conditions for the set $Q_{a}(\mathfrak{m}) \cap \widetilde{\mathfrak{m}}$ to be nonempty}

We will use the notation introduced in Subsection 2.3.

Proposition 14. Suppose that $R(\mathfrak{m}) \cap \widetilde{\mathfrak{m}} \neq \varnothing$ and choose an arbitrary point $x_{0} \in$ $R(\mathfrak{m}) \cap R(\widetilde{\mathfrak{m}})$. Let $\mathfrak{k}^{x_{0}}=\mathfrak{z}(\mathfrak{g})$. The set $Q_{a}(\mathfrak{m}) \cap \widetilde{\mathfrak{m}}$ is nonempty if and only if

the subspace $\mathfrak{k}^{\prime}=(1-\sigma) \mathfrak{k}$ contains regular elements of the Lie algebra $\mathfrak{k}$.

Proof. Let $V$ be some vector subspace of the space $\mathfrak{m}$ for which $V \cap R(\mathfrak{m}) \neq \varnothing$. Put

$$
m_{a}(V)=\min _{x \in V \cap R(\mathfrak{m})} \operatorname{dim}\left(\mathfrak{m}(x) \cap\left(\operatorname{ad}_{a}^{-1} \operatorname{ad} x(\mathfrak{k})\right)\right),
$$

where, recall, $\operatorname{ad}_{a}^{-1} \stackrel{\text { def }}{=}\left(\left.\operatorname{ad} a\right|_{\mathfrak{m}}\right)^{-1}$. By $(12), m_{a}(\mathfrak{m})=r(\mathfrak{m})$ and clearly $m_{a}(V) \geqslant$ $r(\mathfrak{m})$. We have to show that $m_{a}(\widetilde{\mathfrak{m}})=r(\mathfrak{m})$ if and only if condition (21) holds.

We will prove this proposition using the moment map theory and the method proposed in $[\mathrm{Pa}]$. Since $\mathfrak{k}^{x_{0}}=\mathfrak{z}(\mathfrak{g})$, then by the dimension $\operatorname{arguments} \mathfrak{k}^{x}=\mathfrak{z}(\mathfrak{g})$ for all $x \in R(\mathfrak{m})$. For our aim it is convenient to use the moment map constructed in 
our previous paper $[\mathrm{MP}]$. To this end, here we briefly describe the main properties of this moment map [MP, Rem. 3.2].

Consider on the vector space $\mathfrak{m}$ the non-degenerate bilinear form

$$
\beta\left(y_{1}, y_{2}\right)=\left\langle y_{1}, \operatorname{ad}_{a}^{-1}\left(y_{2}\right)\right\rangle, \quad y_{1}, y_{2} \in \mathfrak{m} .
$$

Since the endomorphism ad $\left.a\right|_{\mathfrak{m}}: \mathfrak{m} \rightarrow \mathfrak{m}$ is skew-symmetric (with respect to the form $\langle\cdot, \cdot\rangle$ ), the form $\beta$ is also skew-symmetric. Identifying the tangent space $T_{x} \mathfrak{m}$ with $\mathfrak{m}$ for each $x \in \mathfrak{m}$, we can consider $\beta$ as a symplectic form on $\mathfrak{m}$. Since the Ad-action of $K$ on $\mathfrak{m}$ preserves the form $\beta$, this action of $K$ is Hamiltonian with the $K$-equivariant moment map

$$
\mu^{\beta}: \mathfrak{m} \rightarrow \mathfrak{k}^{*}, \quad \mu^{\beta}(x)(\zeta)=-\frac{1}{2}\left\langle\operatorname{ad}_{a}^{-1}(x),[\zeta, x]\right\rangle, \quad \forall \zeta \in \mathfrak{k}
$$

(see [MP, Rem. 3.2]). In particular, for each $\zeta \in \mathfrak{k}$ the vector field $\zeta_{X}(x)=$ $[\zeta, x] \in T_{x} \mathfrak{m}$ is the Hamiltonian vector field of the function $f_{\zeta}(x)=\mu^{\beta}(x)(\zeta)$ on the manifold $X=\mathfrak{m}$.

Let $x \in \mathfrak{m}, W_{x} \subset T_{x} \mathfrak{m}$ be the tangent space to the $K$-orbit in $\mathfrak{m}$ and let $W_{x}^{\beta \perp}$ be the (skew)orthogonal complement to $W_{x}$ in $T_{x} \mathfrak{m}$ with respect to the form $\beta$. It is easy to see that $W_{x}=\operatorname{ad} x(\mathfrak{k})$ and $W_{x}^{\beta \perp}=\operatorname{ad} a(\mathfrak{m}(x))$, i.e.,

$$
\operatorname{dim}\left(W_{x} \cap W_{x}^{\beta \perp}\right)=\operatorname{dim}(\operatorname{ad} a(\mathfrak{m}(x)) \cap \operatorname{ad} x(\mathfrak{k}))=\operatorname{dim}\left(\mathfrak{m}(x) \cap\left(\operatorname{ad}_{a}^{-1} \operatorname{ad} x(\mathfrak{k})\right)\right) .
$$

However, by the $K$-equivariance of the moment map $\mu^{\beta}, \zeta_{X}(x) \in W_{x} \cap W_{x}^{\beta \perp}$ if and only if $\operatorname{ad}^{*} \zeta(\alpha)=0$, where $\alpha=\mu^{\beta}(x)$ [GS]. In other words, the dimension $\operatorname{dim}\left(W_{x} \cap W_{x}^{\beta \perp}\right)+\operatorname{dim} \mathfrak{z}(\mathfrak{g})$ equals the codimension of the orbit $\operatorname{Ad}^{*}(K) \cdot \alpha$ in $\mathfrak{k}^{*}$ for each $x \in R(\mathfrak{m})\left(\zeta_{X}(x)=0\right.$ if and only if $\left.\zeta \in \mathfrak{k}^{x}=\mathfrak{z}(\mathfrak{g})\right)$.

Identifying the space $\mathfrak{k}^{\mathfrak{k}}$ with its dual $\mathfrak{k}^{*}$ by means of the form $\langle\cdot, \cdot\rangle$, we obtain that

$$
\mu^{\beta}: \mathfrak{m} \rightarrow \mathfrak{k}, \quad \mu^{\beta}(x)=\frac{1}{2}\left[\operatorname{ad}_{a}^{-1}(x), x\right]_{\mathfrak{k}}
$$

and $\operatorname{dim}\left(W_{x} \cap W_{x}^{\beta \perp}\right)=\operatorname{dim} \mathfrak{k}^{\alpha}-\operatorname{dim} \mathfrak{z}(\mathfrak{g})$ if $x \in R(\mathfrak{m})$. Thus

$$
m_{a}(V)=\min _{x \in V \cap R(\mathfrak{m})} \operatorname{dim} \mathfrak{k}^{\alpha(x)}-\operatorname{dim} \mathfrak{z}(\mathfrak{g})=\min _{x \in V} \operatorname{dim} \mathfrak{k}^{\alpha(x)}-\operatorname{dim} \mathfrak{z}(\mathfrak{g}),
$$

where $\alpha(x)=\mu^{\beta}(x) \in \mathfrak{k}$ and $V \cap R(\mathfrak{m}) \neq \varnothing$.

Since $\mathfrak{z}(\mathfrak{g}) \subset \mathfrak{k}$, the space $\mathfrak{m}$ is contained in the maximal semi-simple ideal $\mathfrak{g}_{s}$ of $\mathfrak{g}$. Notice also that $\mu^{\beta}(\widetilde{\mathfrak{m}}) \subset[\mathfrak{g}, \mathfrak{g}]_{\mathfrak{k}} \subset \mathfrak{g}_{s}$ and by $(16) \mu^{\beta}(\widetilde{\mathfrak{m}}) \subset\left[\mathfrak{m}^{\prime}, \widetilde{\mathfrak{m}}\right]_{\mathfrak{k}} \subset\left(\mathfrak{g}^{\prime}\right)_{\mathfrak{k}}=\mathfrak{k}^{\prime}$.

To determine the number $\min _{x \in \widetilde{\mathfrak{m}}} \operatorname{dim} \mathfrak{k}^{\alpha(x)}$ we will show that the image $\mu^{\beta}(\widetilde{\mathfrak{m}})$ contains an open subset in the space $\mathfrak{k}^{\prime} \cap \mathfrak{g}_{s}$. It is easy to calculate that, for any tangent vector $y \in \mathfrak{m}=T_{x_{0}} \mathfrak{m}$,

$$
D_{x_{0}}(y) \stackrel{\text { def }}{=} \mu_{*}^{\beta}\left(x_{0}\right)(y)=\frac{1}{2}\left[\operatorname{ad}_{a}^{-1}\left(x_{0}\right), y\right]_{\mathfrak{k}}+\frac{1}{2}\left[\operatorname{ad}_{a}^{-1}(y), x_{0}\right]_{\mathfrak{k}} .
$$

Taking into account relations (15) and (16) and the inclusion $x_{0} \in \tilde{\mathfrak{m}} \subset \tilde{\mathfrak{g}}$, we obtain that

$$
D_{x_{0}}\left(\mathfrak{m}^{\prime}\right) \subset\left(\left[\operatorname{ad}_{a}^{-1}(\widetilde{\mathfrak{m}}), \mathfrak{m}^{\prime}\right]+\left[\operatorname{ad}_{a}^{-1}\left(\mathfrak{m}^{\prime}\right), \widetilde{\mathfrak{m}}\right]\right)_{\mathfrak{k}} \subset(\widetilde{\mathfrak{g}})_{\mathfrak{k}} \subset \widetilde{\mathfrak{k}}
$$


and, similarly, $D_{x_{0}}(\widetilde{\mathfrak{m}}) \subset\left(\mathfrak{g}^{\prime}\right)_{\mathfrak{k}} \subset \mathfrak{k}^{\prime}$. In other words, $D_{x_{0}}(\mathfrak{m})=D_{x_{0}}\left(\mathfrak{m}^{\prime}\right) \oplus D_{x_{0}}(\widetilde{\mathfrak{m}})$.

The image $\mu_{*}^{\beta}\left(T_{x_{0}} \mathfrak{m}\right) \subset \mathfrak{k}$ of the tangent map of the moment map $\mu^{\beta}$ at $x_{0}$ coincides with the annihilator in $\mathfrak{k}^{*} \simeq \mathfrak{k}$ of the Lie algebra $\mathfrak{k}^{x_{0}}$ of the isotropy group $\left\{k \in K_{0}: \operatorname{Ad} k\left(x_{0}\right)=x_{0}\right\}$ of $x_{0} \in \mathfrak{m}$ [GS]. Since this annihilator coincides with the orthogonal complement of the center $\mathfrak{z}(\mathfrak{g})$ in $\mathfrak{k}$, we obtain that $D_{x_{0}}(\mathfrak{m})=\mathfrak{k} \cap \mathfrak{g}_{s}$. But $\mathfrak{k} \cap \mathfrak{g}_{s}=\left(\mathfrak{k}^{\prime} \cap \mathfrak{g}_{s}\right) \oplus\left(\widetilde{\mathfrak{k}} \cap \mathfrak{g}_{s}\right)$ because $\sigma\left(\mathfrak{g}_{s}\right)=\mathfrak{g}_{s}$. Therefore $\mu_{*}^{\beta}\left(x_{0}\right)(\widetilde{\mathfrak{m}})=\mathfrak{k}^{\prime} \cap \mathfrak{g}_{s}$ and, consequently, the set $\mu^{\beta}(\widetilde{\mathfrak{m}})$ contains some open subset in $\mathfrak{k}^{\prime} \cap \mathfrak{g}_{s}$. Also $\mathfrak{k}^{\alpha}=\mathfrak{k}^{\alpha+z}$ for any $\alpha \in \mathfrak{k}^{\prime}, z \in \mathfrak{z}(\mathfrak{g})$. Therefore

$$
m_{a}(\widetilde{\mathfrak{m}})=\min _{\alpha \in \mathfrak{k}^{\prime} \cap \mathfrak{g}_{s}} \operatorname{dim} \mathfrak{k}^{\alpha}-\operatorname{dim} \mathfrak{z}(\mathfrak{g})=\min _{\alpha \in \mathfrak{k}^{\prime}} \operatorname{dim} \mathfrak{k}^{\alpha}-\operatorname{dim} \mathfrak{z}(\mathfrak{g})
$$

Since $\mathfrak{k}^{x_{0}}=\mathfrak{z}(\mathfrak{g})$ is a commutative algebra, each element of the set $R(\mathfrak{m})$ is a regular element of $\mathfrak{g}$, i.e., $q(\mathfrak{m})=\operatorname{rank} \mathfrak{g}$ and $r(\mathfrak{m})=\operatorname{rank} \mathfrak{g}-\operatorname{dim} \mathfrak{z}(\mathfrak{g})$ (see $(7))$. Also rank $\mathfrak{k}=\operatorname{rank} \mathfrak{g}$ because $\mathfrak{k}=\mathfrak{g}^{a}$. Hence by $(22) m_{a}(\widetilde{\mathfrak{m}})=r(\mathfrak{m})$ if and only if the algebra $\mathfrak{k}^{\prime}$ contains regular elements of the Lie algebra $\mathfrak{k}$.

\subsection{Sufficient conditions for the set $M_{a}(\mathfrak{m}) \cap \widetilde{\mathfrak{m}}$ to be nonempty}

In this subsection we will give a sufficient condition for an open subset $M_{a}(\mathfrak{m}) \cap \widetilde{\mathfrak{m}}$ of $\widetilde{\mathfrak{m}}$ to be nonempty. This condition is motivated by some trick used in the proof of Theorem 3.4 in [BJ3]. Here we will use the notation introduced in Subsection 2.3.

Let $\mathfrak{t}$ be a Cartan subalgebra of $\mathfrak{g}$ containing the element $a$. The complex space $\mathfrak{t}^{\mathbb{C}}$ is a Cartan subalgebra of the reductive complex Lie algebra $\mathfrak{g}^{\mathbb{C}}$. Since $\mathfrak{k}=\mathfrak{g}^{a}$ and the commutative Lie algebra $\mathfrak{t}$ contains $a, \mathfrak{t}^{\mathbb{C}}$ is also a Cartan subalgebra of $\mathfrak{k}^{\mathbb{C}}$. Let $\Delta$ be the root system of $\mathfrak{g}^{\mathbb{C}}$ with respect to $\mathfrak{t}^{\mathbb{C}}$. Denote by $\Delta_{\mathfrak{k}}$ the set of roots in $\Delta$ which vanish identically on $a$. This is the root system of $\left(\mathfrak{k}^{\mathbb{C}}, \mathfrak{t}^{\mathbb{C}}\right)$. Put $\Delta_{\mathfrak{m}}=\Delta \backslash \Delta_{\mathfrak{k}}$. Then we have the direct decompositions

$$
\mathfrak{g}^{\mathbb{C}}=\mathfrak{t}^{\mathbb{C}} \oplus \sum_{\alpha \in \Delta} \mathfrak{g}^{\alpha}, \quad \mathfrak{k}^{\mathbb{C}}=\mathfrak{t}^{\mathbb{C}} \oplus \sum_{\alpha \in \Delta_{\mathfrak{k}}} \mathfrak{g}^{\alpha}, \quad \mathfrak{m}^{\mathbb{C}}=\sum_{\alpha \in \Delta_{\mathfrak{m}}} \mathfrak{g}^{\alpha},
$$

where $\mathfrak{g}^{\alpha}$ is the root space corresponding to the root $\alpha$. We can choose a Weyl basis $\left\{E_{\alpha}, \alpha \in \Delta\right\}$ of $\mathfrak{g}^{\mathbb{C}} \bmod \mathfrak{t}^{\mathbb{C}}$ such that $E_{\alpha} \in \mathfrak{g}^{\alpha}$ for each $\alpha \in \Delta$ and the compact form $\mathfrak{g}$ of $\mathfrak{g}^{\mathbb{C}}$ is spanned by $\mathfrak{t}$ and the vectors $X_{\alpha}=\left(E_{\alpha}-E_{-\alpha}\right), Y_{\alpha}=i\left(E_{\alpha}+E_{-\alpha}\right)$, $\alpha \in \Delta$.

Proposition 15. Suppose that $R(\mathfrak{m}) \cap \widetilde{\mathfrak{m}} \neq \varnothing$ and $\mathfrak{k}^{x}=\mathfrak{z}(\mathfrak{g})$ for all $x \in R(\mathfrak{m})$. Assume that there exists a system of simple roots $\pi$ of $\Delta$ such that $\pi \subset \Delta_{\mathfrak{m}}$ and some vector

$$
x_{\pi}=\sum_{\alpha \in \pi} c_{\alpha} E_{-\alpha}+\sum_{\beta \in \Delta_{\mathfrak{m}}^{+}} d_{\beta} E_{\beta}, \quad c_{\alpha} \in \mathbb{C} \backslash\{0\}, \alpha \in \pi, d_{\beta} \in \mathbb{C}, \beta \in \Delta_{\mathfrak{m}}^{+},
$$

where $\Delta^{+}$is the system of positive roots of $\Delta$ determined by $\pi$ and $\Delta_{\mathfrak{m}}^{+}=\Delta_{\mathfrak{m}} \cap \Delta^{+}$, belongs to the space $\widetilde{\mathfrak{m}}^{\mathbb{C}} \subset \mathfrak{m}^{\mathbb{C}}$. Then the set $M_{a}(\mathfrak{m}) \cap \widetilde{\mathfrak{m}}$ is nonempty.

Proof. Since for $x \in R(\mathfrak{m})$ the algebra $\mathfrak{k}^{x}=\mathfrak{z}(\mathfrak{g})$ is commutative, the centralizer $\mathfrak{g}^{x}$ is a Cartan subalgebra of $\mathfrak{g}$ (see $(5)$ ), i.e., $q(\mathfrak{m})=$ rank $\mathfrak{g}$. 
The element $e_{-}=\sum_{\alpha \in \pi} c_{\alpha} E_{-\alpha}$ is a principal nilpotent element of the reductive complex Lie algebra $\mathfrak{g}^{\mathbb{C}}[\mathrm{Ko}]$. Since $a \in \mathfrak{t}^{\mathbb{C}}$, for each $\lambda \in \mathbb{C}$ the vector $x_{\pi}+\lambda a$ is an element of the affine subspace $e_{-}+\mathfrak{b}$, where $\mathfrak{b}=\mathfrak{t}^{\mathbb{C}} \oplus \sum_{\beta \in \Delta^{+}} \mathfrak{g}^{\beta}$ is a Borel subalgebra of $\mathfrak{g}^{\mathbb{C}}$. Then by [Ko, Lem. 10] $\operatorname{dim}_{\mathbb{C}}\left(\mathfrak{g}^{\mathbb{C}}\right)^{x_{\pi}+\lambda a}=\operatorname{rank} \mathfrak{g}^{\mathbb{C}}$. But $q(\mathfrak{m})=$ rank $\mathfrak{g}^{\mathbb{C}}$. Thus $x_{\pi} \in M_{a}\left(\mathfrak{m}^{\mathbb{C}}\right) \cap \widetilde{\mathfrak{m}}^{\mathbb{C}}$ (see the proof of Lemma 8). Since $M_{a}\left(\mathfrak{m}^{\mathbb{C}}\right) \cap \widetilde{\mathfrak{m}}^{\mathbb{C}}$ is a nonempty Zariski open subset of $\widetilde{\mathfrak{m}}^{\mathbb{C}}$, its intersection with the real form $\widetilde{\mathfrak{m}} \subset \widetilde{\mathfrak{m}}^{\mathbb{C}}$ is nonempty. Taking into account that $M_{a}\left(\mathfrak{m}^{\mathbb{C}}\right) \cap \widetilde{\mathfrak{m}}=M_{a}(\mathfrak{m}) \cap \widetilde{\mathfrak{m}}$ we complete the proof.

\subsection{Integrable geodesic flows on $\mathrm{SO}(n) /\left(\mathrm{SO}\left(n_{1}\right) \times \cdots \times \mathrm{SO}\left(n_{p}\right)\right)$}

In this subsection we show that the conditions of Theorem 10 hold for the homogeneous space $\mathrm{SO}(n) /\left(\mathrm{SO}\left(n_{1}\right) \times \cdots \times \mathrm{SO}\left(n_{p}\right)\right), n_{1}+\cdots+n_{p}=n$. The case $p=2$ is not interesting for us from the point of view of integrability because in this case the considered homogeneous space $\widetilde{G} / \widetilde{K}$ is a symmetric space and, consequently, all $\widetilde{G}$-invariant Hamiltonian flows on $T(\widetilde{G} / \widetilde{K})$ are integrable [Mi], [My2].

Consider the symmetric space $G / \widetilde{G}=\mathrm{U}(n) / \mathrm{SO}(n)$, where $n \geqslant 4$, with the involution $\sigma$ on the Lie algebra of skew-hermitian matrices $\mathfrak{g}=\mathfrak{u}(n)$ determined by the complex conjugation. Then the Lie algebra $\widetilde{\mathfrak{g}}=(1+\sigma) \mathfrak{g}$ is the Lie algebra $\mathfrak{s o}(n)$ of all real skew-symmetric $n \times n$ matrices. The space $\mathfrak{g}^{\prime}=(1-\sigma) \mathfrak{g}$ coincides with the set $i \operatorname{sym}(n)$, where $\operatorname{sym}(n)$ is the space of all real symmetric $n \times n$ matrices.

Fix some element $a \in \mathfrak{g}^{\prime}, a=\operatorname{diag}\left(i \lambda_{1}, \ldots, i \lambda_{1}, i \lambda_{2}, \ldots, i \lambda_{2}, \ldots, i \lambda_{p}, \ldots, i \lambda_{p}\right)$, where all real numbers $\lambda_{1}, \ldots, \lambda_{p}$ are pairwise different and the multiplicity of each $i \lambda_{j}$ is equal to $n_{j} \geqslant 1, n_{1}+\cdots+n_{p}=n$. Without loss of generality (to simplify calculations) we may assume that $1 \leqslant n_{1} \leqslant n_{2} \leqslant \cdots \leqslant n_{p}<n$.

It is clear that the Lie algebra $\mathfrak{k}=\mathfrak{g}^{a}$ is the Lie algebra $\mathfrak{u}\left(n_{1}\right) \oplus \cdots \oplus \mathfrak{u}\left(n_{p}\right)$ (with the standard block-diagonal embedding) and $\widetilde{\mathfrak{k}}$ is the real part of this Lie algebra, i.e., $\widetilde{\mathfrak{k}}$ coincides with $\mathfrak{s o}\left(n_{1}\right) \oplus \cdots \oplus \mathfrak{s o}\left(n_{p}\right)(\mathfrak{s o}(1)=0)$. In this case, $\widetilde{G} / \widetilde{K}=\mathrm{SO}(n) /\left(\mathrm{SO}\left(n_{1}\right) \times \cdots \times \mathrm{SO}\left(n_{p}\right)\right)$.

Putting $\langle X, Y\rangle=-\operatorname{Tr} X Y$ (using the trace-form) we define an invariant scalar product on $\mathfrak{g}$. To describe the space $\mathfrak{m}=\mathfrak{k}^{\perp}$ consider any matrix $X \in \mathfrak{g}$ as a block-matrix consisting of rectangle elements $X^{k, l}$, which are rectangle complex $n_{k} \times n_{l}$ matrices, $1 \leqslant k, l \leqslant p$. It is clear that $\left(\overline{X^{k, l}}\right)^{t}=-X^{l, k}$ and therefore any element of $\mathfrak{u}(n)$ is defined by its blocks $X^{k, l}$ with $k \leqslant l$. As a space, the Lie algebra $\mathfrak{g}=\mathfrak{u}(n)$ is a direct sum of its block-type subspaces $V^{k, l}, 1 \leqslant k \leqslant l \leqslant p$. In this notation the Lie subalgebra $\mathfrak{k}$ is the direct sum $\sum_{j=1}^{p} V^{j, j}$ and $\mathfrak{m}=\sum_{1 \leqslant k<l \leqslant p} V^{k, l}$. We will denote the corresponding to $X^{k, l}$ element of the space $V^{k, l}$ by $\varphi\left(X^{k, l}\right)$. Each subspace $V^{k, l}$ is an $\mathfrak{k}$-module, i.e., $\left[V^{k, l}, \mathfrak{k}\right] \subset V^{k, l}$.

First of all consider the simplest case when $p=2$. In this case $G / K=$ $\mathrm{U}\left(n_{1}+n_{2}\right) /\left(\mathrm{U}\left(n_{1}\right) \times \mathrm{U}\left(n_{2}\right)\right)$ is a Hermitian symmetric space. Therefore there exists a Cartan subspace $\mathfrak{a}$ in $V^{1,2}=\mathfrak{m}$ (a maximal commutative subspace in $V^{1,2}$ ) consisting of real matrices (belonging to $\mathfrak{s o}(n)$ ) [He, Chap. X, Sec. 2.1]. This $n_{1^{-}}$ dimensional Cartan subspace $\mathfrak{a}$ can be described by the "diagonal" matrices $X^{1,2}$ in which all entries vanish with the exception of $n_{1}$ entries $X_{j, j}^{1,2}, j=1, \ldots, n_{1} \leqslant n_{2}$, which are arbitrary real numbers. Then the centralizer $\mathfrak{k}^{x_{0}}$ of a regular element (of the Cartan subspace) $x_{0}=\varphi\left(X_{*}^{1,2}\right) \in \widetilde{\mathfrak{m}} \subset V^{1,2}$ in $\mathfrak{u}\left(n_{1}\right) \oplus \mathfrak{u}\left(n_{2}\right)=V^{1,1} \oplus V^{2,2}$ is a 
direct sum $\mathfrak{t}_{*} \oplus \mathfrak{c}_{*}$, where $\mathfrak{c}_{*} \simeq \mathfrak{u}\left(n_{2}-n_{1}\right)$ and $\mathfrak{t}_{*}$ is a commutative algebra of dimension $n_{1}$ consisting of diagonal matrices $\operatorname{diag}\left(i x_{1}, \ldots, i x_{n_{1}}, i x_{1}, \ldots, i x_{n_{1}}, 0, \ldots, 0\right)$, $x_{j} \in \mathbb{R}$. Notice that the maximal semisimple ideal of $\mathfrak{c}_{*}$ coincides with the maximal semisimple ideal of the centralizer of $\mathfrak{t}_{*}$ in $\mathfrak{u}\left(n_{1}\right) \oplus \mathfrak{u}\left(n_{2}\right)$.

Suppose now that $p \geqslant 3$. We will attempt to describe the centralizer $\mathfrak{k}^{x_{0}}$ of some generic element $x_{0} \in \widetilde{\mathfrak{m}}$ constructing this element. To simplify our calculations notice that each space $V^{k, k} \oplus V^{l, l} \oplus V^{k, l}, k<l$ is a Lie subalgebra of $\mathfrak{u}(n)$ isomorphic to $\mathfrak{u}\left(n_{k}+n_{l}\right), V^{k, k} \oplus V^{l, l} \simeq \mathfrak{u}\left(n_{k}\right) \oplus \mathfrak{u}\left(n_{l}\right)$ and $\left[V^{k, l}, V^{k, l}\right] \subset V^{k, k} \oplus V^{l, l}$. But $\mathrm{U}\left(n_{k}+n_{l}\right) /\left(\mathrm{U}\left(n_{k}\right) \times \mathrm{U}\left(n_{l}\right)\right)$ is a Hermitian symmetric space and therefore we can use our calculations for the case $p=2$. Since each subspace $V^{k, l}$ is a $\mathfrak{k}$-module, we will construct the element $x_{0}$ selecting step by step its $V^{k, l}$-entries. For our aim it is enough to consider the submodule $\sum_{j=1}^{p-1} V^{j, j+1} \oplus \sum_{j=1}^{p-2} V^{j, p}$ of $\mathfrak{m}$. Choosing in each $\mathfrak{k}$-module $V^{j, j+1}$ of the first component the "diagonal" element $\varphi\left(X_{*}^{j, j+1}\right)$ as above, we obtain that their common isotropy algebra is the direct sum $\mathfrak{t}_{*} \oplus \mathfrak{c}_{*}$, where $\mathfrak{c}_{*} \simeq \mathfrak{u}\left(n_{p}-n_{p-1}\right)$ and $\mathfrak{t}_{*}$ is of commutative algebra of dimension $n_{p-1}$, consisting of the diagonal matrices $\operatorname{diag}\left(i x_{1}, \ldots, i x_{n_{1}}, i x_{1}, \ldots, i x_{n_{2}}, \ldots\right.$, $\left.i x_{1}, \ldots, i x_{n_{p-1}}, i x_{1}, \ldots, i x_{n_{p-1}}, 0, \ldots, 0\right), x_{j} \in \mathbb{R}, 1 \leqslant j \leqslant n_{p-1}$. Notice that the maximal semisimple ideal of $\mathfrak{c}_{*}$ coincides with the maximal semisimple ideal of the centralizer of $\mathfrak{t}_{*}$ in $\mathfrak{k}$. Now we consider $V=\sum_{j=1}^{p-2} V^{j, p}$ as a $\mathfrak{t}_{*} \oplus \mathfrak{c}_{*}$-module (not as a k-module) of complex dimension $N_{2} \times n_{p}, N_{2}=n_{1}+\cdots+n_{p-2}$. Then $V$ is the direct sum of $\mathfrak{t}_{*} \oplus \mathfrak{c}_{*}$-modules $V^{(1)} \oplus V^{(2)}, V^{(1)} \perp V^{(2)}$, where $V^{(1)}$ (of complex dimension $N_{2} \times n_{p-1}$ ) is a trivial $\mathfrak{c}_{*}$-module. Therefore the isotropy subalgebra of a real generic point from $V^{(1)}$ is the algebra $\mathfrak{t}_{* *} \oplus \mathfrak{c}_{*}$, where $\mathfrak{t}_{* *} \subset \mathfrak{t}_{*}$ is the one-dimensional subalgebra consisting of the elements of $\mathfrak{t}_{*}$ such that $x_{1}=x_{2}=\ldots=x_{n_{p-1}}=\lambda$. Considering the module $V^{(2)}$ as the space $M_{N_{2}, n_{p}-n_{p-1}}$ of complex $N_{2} \times\left(n_{p}-n_{p-1}\right)$ matrices, the ad-representation of $\mathfrak{t}_{* *} \oplus \mathfrak{c}_{*}$ in $V^{(2)}$ is described as follows: $\operatorname{ad}(\lambda, A)(B)=i \lambda B-B A$, where $(\lambda, A) \in \mathbb{R} \oplus \mathfrak{u}\left(n_{p}-n_{p-1}\right) \simeq \mathfrak{t}_{* *} \oplus \mathfrak{c}_{*}$ and $B \in M_{N_{2}, n_{p}-n_{p-1}} \simeq V^{(2)}$.

If the number of rows $N_{2}=n_{1}+\cdots+n_{p-2}$ in $B$ is not less than the number of columns $\left(n_{p}-n_{p-1}\right)$, then for any real matrix $B \in M_{N_{2}, n_{p}-n_{p-1}}$ of the maximal rank we have $i \lambda B-B A=0$ if and only if $A=i \lambda$ (is a scalar matrix). Therefore in this case $\mathfrak{k}^{x_{0}}=\mathfrak{z}(\mathfrak{g})$ for some (real) matrix $x_{0} \in \widetilde{\mathfrak{m}}$.

If $N_{2}<n_{p}-n_{p-1}$, then for the real matrix $B$ in which all entries vanish with the exception of $N_{2}$ entries $B_{j, j}=1, j=1, \ldots, N_{2}$, we obtain that $i \lambda B-B A=0$ if and only if $A$ is an element of a Lie algebra isomorphic to $\mathfrak{u}(1) \oplus \mathfrak{u}\left(n_{p}-n_{p-1}-N_{2}\right)$, i.e., for some (real) matrix $x_{0} \in \widetilde{\mathfrak{m}}$ we have $\operatorname{dim} \mathfrak{k}^{x_{0}}=1+\operatorname{dim} \mathfrak{u}\left(n_{p}-n_{p-1}-N_{2}\right)$.

Suppose now that $p \geqslant 3$ and $n_{1}+\cdots+n_{p-2} \geqslant n_{p}-n_{p-1}$. We showed above that for some element $x_{0} \in \widetilde{\mathfrak{m}}$ its centralizer $\mathfrak{k}^{x_{0}}$ is the one-dimensional center $\mathfrak{z}(\mathfrak{g})$ of $\mathfrak{g}=\mathfrak{u}(n)$. Since $\mathfrak{z}(\mathfrak{g}) \subset \mathfrak{k}^{x}$ for each $x \in \mathfrak{m}$, we have that $\operatorname{dim} \mathfrak{k}^{x_{0}}=p(\mathfrak{m})$ and $\operatorname{dim} \widetilde{\mathfrak{k}}^{x_{0}}=p(\widetilde{\mathfrak{m}})$. Clearly these two properties hold for any points $x \in \widetilde{\mathfrak{m}}$ which are sufficiently closed to $x_{0}$. Therefore we can suppose without losing generality that $x_{0} \in R(\widetilde{\mathfrak{m}})$, i.e., $\operatorname{dim} \widetilde{\mathfrak{g}}^{x_{0}}=q(\widetilde{\mathfrak{m}})$. But $q(\widetilde{\mathfrak{m}})=\operatorname{rank}(\widetilde{\mathfrak{g}})$ because the algebra $\widetilde{\mathfrak{k}}^{x_{0}} \subset \mathfrak{z}(\mathfrak{g})$ is commutative (see $\left.(5)\right)$. Taking into account that any regular element of the Lie algebra $\widetilde{\mathfrak{g}}=\mathfrak{s o}(n)$ is regular in $\mathfrak{g}=\mathfrak{u}(n)$, we obtain that $x_{0} \in R(\mathfrak{m})$. Thus $x_{0} \in R(\mathfrak{m}) \cap R(\widetilde{\mathfrak{m}})$ and $\mathfrak{k}^{x_{0}}=\mathfrak{z}(\mathfrak{g})$.

Now we can use Proposition 14 to prove that $Q_{a}(\mathfrak{m}) \cap \widetilde{\mathfrak{m}} \neq \varnothing$. Indeed, since the 
space $\mathfrak{k}^{\prime}=(1-\sigma) \mathfrak{k}=i \operatorname{sym}\left(n_{1}\right) \oplus \cdots \oplus i \operatorname{sym}\left(n_{p}\right)$ contains regular elements of the Lie algebra $\mathfrak{k}=\mathfrak{u}\left(n_{1}\right) \oplus \cdots \oplus \mathfrak{u}\left(n_{p}\right)$, by this proposition $Q_{a}(\mathfrak{m}) \cap \widetilde{\mathfrak{m}} \neq \varnothing$.

Our element $a \in \mathfrak{u}(n)$ is a diagonal matrix $\operatorname{diag}\left(a_{1}, \ldots, a_{n}\right), a_{j} \in i \mathbb{R}$ and is contained in the Cartan subalgebra $\mathfrak{t}$ of $\mathfrak{u}(n)$ consisting of matrices $h=\operatorname{diag}\left(h_{1}, \ldots, h_{n}\right)$, $h_{j} \in i \mathbb{R}$. The set $\Delta=\left\{\varepsilon_{j k}=\varepsilon_{j}-\varepsilon_{k}, j \neq k ; j, k=1, \ldots, n\right\}, \varepsilon_{j}(h)=h_{j}$, is the standard system of roots of $\mathfrak{g}^{\mathbb{C}}$ with respect to $\mathfrak{t}^{\mathbb{C}}$. The algebra $\mathfrak{t}$ and vectors $X_{j k}=E_{\varepsilon_{j k}}-E_{\varepsilon_{k j}}, Y_{j k}=i E_{\varepsilon_{j k}}+i E_{\varepsilon_{k j}}$ span $\mathfrak{g}$ (by $E_{\varepsilon_{j k}}$ we denote the matrix whose only nonzero element is 1 located at the position $j k$ ). To prove that $M_{a}(\mathfrak{m}) \cap \widetilde{\mathfrak{m}} \neq \varnothing$ we will use the following observation (see [BJ2]): Since $n_{p} \leqslant n_{1}+\cdots+n_{p-1}$, there exists a permutation $p$ of $\{1,2, \ldots, n\}$ such that $a_{p(j)} \neq a_{p(j+1)}, j=1, \ldots, n-1$. In other words, $\left(\varepsilon_{j}-\varepsilon_{j+1}\right)(\hat{a}) \neq 0$ for each $j=1, \ldots, n-1$, where $\hat{a}=\operatorname{diag}\left(a_{p(1)}, a_{p(2)}, \ldots, a_{p(n)}\right)$. As the Weyl group of $(\mathfrak{g}, \mathfrak{t})$ is the permutation group of $n$ elements, there exists the system of simple roots $\pi$ of $\Delta$ such that $\alpha(a) \neq 0$ for each $\alpha \in \pi$. Since the element $\sum_{\alpha \in \pi}\left(E_{\alpha}-E_{-\alpha}\right)$ (real skew-symmetric matrix) belongs to the space $\widetilde{\mathfrak{m}}$, then by Proposition 15 , $M_{a}(\mathfrak{m}) \cap \widetilde{\mathfrak{m}} \neq \varnothing$, and by $(20), O^{\mathrm{Kr}}(\mathfrak{m}) \cap \widetilde{\mathfrak{m}} \neq \varnothing$.

Suppose now that $p \geqslant 3$ and $n_{1}+\cdots+n_{p-1}<n_{p}$. Put $N_{1}=n_{1}+\cdots+n_{p-1}$. In this case, since the last component $\mathfrak{k}_{p} \simeq \mathfrak{u}\left(n_{p}\right)$ of $\mathfrak{k}$ is dominant in $\mathfrak{k}$, the calculation problem can be reduced to the previous case with $n_{p}=n_{1}+\cdots+n_{p-1}$. To this end we consider the representation of the Lie group $K_{p} \subset K$ with the Lie algebra

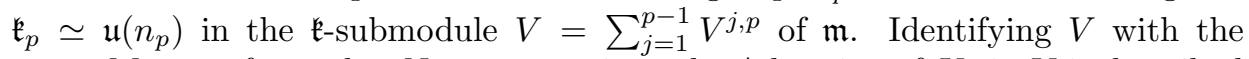
space $M_{N_{1}, n_{p}}$ of complex $N_{1} \times n_{p}$ matrices, the Ad-action of $K_{p}$ in $V$ is described as follows: $k \cdot B=B k^{-1}$, where $k \in K_{p}=\mathrm{U}\left(n_{p}\right), B \in M_{N_{1}, n_{p}}$. Since the number of rows $N_{1}$ in $B$ is less than its number of columns $n_{p}$, then the $\operatorname{Ad}\left(K_{p}\right)$ orbit of $B$ in $V$ contains a matrix in which the last $n_{p}-N_{1}$ columns vanish. In other words, each element of $\mathfrak{m}$ is $\operatorname{Ad}(K)$-conjugated to some element of the first component $\mathfrak{u}\left(2 N_{1}\right)$ of the Lie algebra $\mathfrak{u}\left(2 N_{1}\right) \oplus \mathfrak{u}\left(n_{p}-N_{1}\right) \subset \mathfrak{u}(n)$ and, consequently, $\operatorname{dim} \mathfrak{k}^{x} \geqslant 1+\operatorname{dim} \mathfrak{u}\left(n_{p}-N_{1}\right)(\operatorname{dim} \mathfrak{z}(\mathfrak{g})=1)$ for any $x \in R(\mathfrak{m})$. But we showed above that for some element $x_{0} \in \widetilde{\mathfrak{m}}$ we have an equality, i.e., this element belongs to the set $R(\mathfrak{m}) \cap \widetilde{\mathfrak{m}}$. Since the set $R(\widetilde{\mathfrak{m}})$ is Zariski open in $\widetilde{\mathfrak{m}}$, we can suppose without losing generality that $x_{0} \in R(\mathfrak{m}) \cap R(\widetilde{\mathfrak{m}})$ and $\mathfrak{k}^{x_{0}}=\mathfrak{z}(\mathfrak{g}) \oplus \mathfrak{u}\left(n_{p}-N_{1}\right)$. Taking into account that $\mathfrak{u}\left(2 N_{1}\right) \oplus \mathfrak{u}\left(n_{p}-N_{1}\right)$ is a maximal subalgebra of $\mathfrak{u}(n)$ (the pair $\left(\mathfrak{u}(n), \mathfrak{u}\left(2 N_{1}\right) \oplus \mathfrak{u}\left(n_{p}-N_{1}\right)\right)$ is a symmetric pair), we obtain that the centralizer $\mathfrak{g}_{0}$ of $\mathfrak{k}^{x_{0}}$ in $\mathfrak{g}$ is the algebra $\mathfrak{u}\left(2 N_{1}\right) \oplus \mathfrak{z}(\mathfrak{g})$ containing the element $a$. Now applying Theorem 13 and Remark 12 we reduce our problem to the case when $n_{p}=n_{1}+\cdots+n_{p-1}$. In other words, $O^{\mathrm{Kr}}(\mathfrak{m}) \cap \widetilde{\mathfrak{m}} \neq \varnothing$, i.e., the condition of Theorem 10 holds.

Thus the new proof of Theorem 4 in [DGJ1] is obtained, i.e., the following theorem is proved.

Theorem 16 ([DGJ1]). We retain the notation of Theorem 10. Let $G=\mathrm{U}(n)$, $\widetilde{G}=\mathrm{SO}(n)$ and $\widetilde{K}=\mathrm{SO}\left(n_{1}\right) \times \cdots \times \mathrm{SO}\left(n_{p}\right), n=n_{1}+\cdots+n_{p}$, with the standard embedding of $\widetilde{K} \subset \widetilde{G} \subset G$. The set of functions $\mathcal{F}(\mathfrak{g}, \widetilde{\mathfrak{m}})$ is a complete involutive subset of the Poisson algebra $\left(A^{\widetilde{G}}, \widetilde{\eta}^{\text {can }}\right)$. The set of functions $\mathcal{H}\left(\widetilde{\mathfrak{g}}^{*}\right) \cup \mathcal{F}(\mathfrak{g}, \widetilde{\mathfrak{m}})$ is a complete involutive subset of the algebra $\left(\mathcal{E}(T(\widetilde{G} / \widetilde{K})), \widetilde{\eta}^{\text {can }}\right)$. The Hamiltonian flow with the Hamiltonian function $\widetilde{H}_{a, b}$ on $T(\widetilde{G} / \widetilde{K})$ is completely integrable by 
means of real-analytic integrals $\mathcal{H}\left(\widetilde{\mathfrak{g}}^{*}\right) \cup \mathcal{F}(\mathfrak{g}, \widetilde{\mathfrak{m}})$.

Remark 17. Let $\widetilde{\mathfrak{g}}=\mathfrak{s o}(n)$ and let $\widetilde{\mathfrak{k}}=\mathfrak{s o}\left(n_{1}\right) \oplus \cdots \oplus \mathfrak{s o}\left(n_{p}\right)$. Suppose that $n_{p} \leqslant n_{1}+\cdots+n_{p-1}$. Then the set $\widetilde{\mathfrak{m}}=\widetilde{\mathfrak{k}}^{\perp}(\widetilde{\mathfrak{g}}=\widetilde{\mathfrak{m}} \oplus \widetilde{\mathfrak{k}})$ contains regular elements of the Lie algebra $\mathfrak{s o}(n)$ (see, for example, the proof of Theorem 16 above). In [DGJ2] Dragović, Gajić and Jovanović remarked that the first part of the proof of their Theorem 4 published in [DGJ1] is not complete. Namely, relation (29) of [DGJ1, Rem.1], i.e., , the completeness of some family of integrals, holds for all elements from some open dense subset $O$ of $\mathfrak{s o}(n)$ (for the so-called generic elements). However, although $\widetilde{\mathfrak{m}}$ contains regular elements of $\mathfrak{s o}(n)$, relation (29) holds on $\widetilde{\mathfrak{m}}$, i.e., $O \cap \widetilde{\mathfrak{m}} \neq \varnothing$, if and only if all $n_{1}, \ldots, n_{p}$ are $\leqslant 2$ (see [DGJ2, p. 1287]). Therefore, relation (36) of [DGJ1], and, consequently, the first part of the proof of Theorem 4 [DGJ1] needs an additional argumentation in the case when $n_{p} \geqslant 3$.

\section{References}

[DGJ1] V. Dragović, B. Gajić, B. Jovanović, Singular Manakov flows and geodesic flows on homogeneous spaces of $\mathrm{SO}(N)$, Transform. Groups 14 (2009), no. 3, 513-530.

[DGJ2] V. Dragović, B. Gajić, B. Jovanović, Systems of Hess-Appelrot type and Zhukovskii property, Internat. J. Geom. Methods in Modern Physics 6 (2009), no. 8, 1253-1304.

[ВJ1] А. В. Болсинов, Б. Йованович, Интегрируемые геодезические потоки на однородных пространствах, Матем. сб. 192 (2001), nо. 7, 21-40. Engl. transl.: A. V. Bolsinov, B. Jovanovich, Integrable geodesic flows on homogeneous spaces, Sb. Math. 192 (2001), no. 7-8, 951-968.

[BJ2] A. V. Bolsinov, B. Jovanović, Non-commutative integrability, moment map and geodesic flows, Ann. Global Anal. Geom. 23 (2003), no. 4, 305-322.

[BJ3] A. V. Bolsinov, B. Jovanović, Complete involutive algebras of functions on cotangent bundles of homogeneous spaces, Math. Z., 246 (2004), n. 1-2, 213-236.

[BJ4] A. V. Bolsinov, B. Jovanovic, Integrable geodesic flows on Riemannian manifolds: construction and obstructions, in: Contemporary Geometry and Related Topics, World Sci. Publ., River Edge, NJ, 2004, pp. 57-103.

[Во] А. В. Болсинов, Согласованнье скобки Пуассона на алгебрах Ли и полнота семейств функиий в инволющии, Изв. АН СССР, Сер. матем. 55 (1991), no. 1, 68-92. Engl. transl.: A. V. Bolsinov, Compatible Poisson brackets on Lie algebras and completeness of families of functions in involution, Math. USSRIzvestiya 38 (1992), no. 1, 69-90.

[Br] А. В. Браилов, Построение вполне интегрируемых геодезических потоков на компактных симметрических пространствах, Изв. АН СССР, Cер. матем. 50 (1986), no. 2, 661-674. Engl. transl.: A. V. Brailov, Construction of completely integrable geodesic flows on compact symmetric spaces, Math. USSRIzvestiya 29 (1987), no. 1, 19-31.

[Bou2] N. Bourbaki, Groupes et algèbres de Lie, VII, VIII, Éléments de mathématique, Hermann, Paris, 1975.

[GS] V. Guillemin, S. Sternberg, On collective complete integrability according to the method of Thimm, Ergod. Theory and Dynam. Syst. 3 (1983), no. 2, 219-230. 
[He] S. Helgason, Differential Geometry, Lie Groups, and Symmetric Spaces, Academic Press, New York, 1978.

[Ko] B. Kostant, Lie group representation in polynomial rings, Amer. J. Math. 85 (1963), no. 3, 327-404.

[Mа] С. В. Манаков, Замечание об интегрировании уравнений Эйлера динамики $n$-мерного твёрдого тела, Функц. анализ и его прил. 10 (1976), вып. 4, 9394. Engl. transl.: S. V. Manakov, Note on the integration of Euler's equations of the dynamics of an n-dimensional rigid body, Funct. Anal. Appl., 10 (1977), no. 4, 328-329.

[MF1] А. С. Мищенко, А. Т. Фоменко, Уравнения Эйлера на конечномерных групnax Ли, Изв. АН СССР, Сер. матем. 42 (1978), no. 2, 396-415. Engl. transl.: A. S. Mishchenko, A. T. Fomenko, Euler equations on finite-dimensional Lie groups, Math. USSR-Izv. 12 (1978), no. 2, 371-389.

[MF2] А. С. Мищенко, А. Т. Фоменко, Обобщённый метод Лиувилля интегрирования гамильтоновых систем, Функц. анализ и его прил. 12 (1978), вып. 2, 46-56. Engl. transl.: A. S. Mishchenko, A. T. Fomenko, Generalized Liouville method of integration of Hamiltonian systems, Funct. Anal. Appl. 12 (1978), no. 2, 113-121.

[Mi] А. С. Мищенко, Интегрирование геодезических потоков на симметрических пространствах, Матем. заметки 31 (1982), no. 2, 257-262. Engl. transl.: A. S. Mishchenko, Integration of geodesic flows on symmetric spaces, Math. Notes 31 (1982), no. 1-2, 132-134.

[MS] I. V. Mykytyuk, A. M. Stepin, Classification of almost spherical pairs of compact simple Lie groups, Banach Center Publications 51 (2000), 231-241.

[MP] I. V. Mykytyuk, A. Panasyuk, Bi-Poisson structures and integrability of geodesic flows on homogeneous spaces, Transform. Groups 9 (2004), no. 3, 289-308.

[Mу1] И. В. Микитюк, Однородные пространства с интегрируемыми G-инвариантными гамильтоновыми потоками, Изв. АН СССР, Сер. матем. 47 (1983), no. 6, 1248-1262. Engl. transl.: I. V. Mikityuk, Homogeneous spaces with integrable G-invariant Hamiltonian flows, Math. USSR-Izvestiya 23 (1984), no. 3, 511-523.

[Mу2] И. В. Микитюк, Об интегрируемости инвариантных гамильтоновых систем с однородными конфигурационными пространствами, Матем. сб. 129(171) (1986), no. 4, 514-534. Engl. transl.: I. V. Mikityuk, On the integrability of invariant Hamiltonian systems with homogeneous configuration spaces, Math. USSR-Sbornik 57 (1987), no. 2, 527-546.

[My3] I. V. Mykytyuk, Actions of Borel subgroups on homogeneous spaces of reductive complex Lie groups and integrability, Compositio Math. 127 (2001), no. 1, 55-67.

[Pa] A. Panasyuk, Projections of Jordan bi-Poisson structures that are Kronecker, diagonal actions, and the classical Gaudin systems, J. Geom. Phys. 47 (2003), 379-397.

[TF] В. В. Трофимов, А. Т. Фоменко, Алгебра и геометрия интегрируемых гамильтоновых дифференциальньх уравнений, Фекториал, М., 1995. [V. V. Trofimov, A. T. Fomenko, Algebra and geometry of integrable Hamiltonian differential equations, Faktorial, Moscow, 1995 (Russian).] 
Open Access This article is distributed under the terms of the Creative Commons Attribution 4.0 International License (http://creativecommons.org /licenses/by/4.0/), which permits unrestricted use, distribution, and reproduction in any medium, provided you give appropriate credit to the original author(s) and the source, provide a link to the Creative Commons license, and indicate if changes were made. 